\title{
Review \\ Status Quo in Data Availability and Predictive Models of Nano-Mixture Toxicity
}

\author{
Tung X. Trinh ${ }^{1,2}$ and Jongwoon Kim ${ }^{1, *(1)}$ \\ 1 Chemical Safety Research Center, Korea Research Institute of Chemical Technology (KRICT), \\ Daejeon 34114, Korea; trinhxt@krict.re.kr \\ 2 Department of Chemistry, College of Natural Sciences, Hanyang University, Seoul 04763, Korea \\ * Correspondence: jkim@krict.re.kr; Tel.: +82-(0)42-860-7482
}

\section{check for}

updates

Citation: Trinh, T.X.; Kim, J. Status Quo in Data Availability and Predictive Models of Nano-Mixture Toxicity. Nanomaterials 2021, 11, 124. https://doi.org/10.3390/nano11010124

Received: 18 December 2020

Accepted: 4 January 2021

Published: 7 January 2021

Publisher's Note: MDPI stays neutral with regard to jurisdictional clai$\mathrm{ms}$ in published maps and institutional affiliations.

Copyright: $\odot 2021$ by the authors. Licensee MDPI, Basel, Switzerland. This article is an open access article distributed under the terms and conditions of the Creative Commons Attribution (CC BY) license (https:// creativecommons.org/licenses/by/ $4.0 /)$.

\begin{abstract}
Co-exposure of nanomaterials and chemicals can cause mixture toxicity effects to living organisms. Predictive models might help to reduce the intensive laboratory experiments required for determining the toxicity of the mixtures. Previously, concentration addition (CA), independent action (IA), and quantitative structure-activity relationship (QSAR)-based models were successfully applied to mixtures of organic chemicals. However, there were few studies concerning predictive models for toxicity of nano-mixtures before June 2020. Previous reviews provided comprehensive knowledge of computational models and mechanisms for chemical mixture toxicity. There is a gap in the reviewing of datasets and predictive models, which might cause obstacles in the toxicity assessment of nano-mixtures by using in silico approach. In this review, we collected 183 studies of nano-mixture toxicity and curated data to investigate the current data and model availability and gap and to derive research challenges to facilitate further experimental studies for data gap filling and the development of predictive models.
\end{abstract}

Keywords: nano-mixture; toxicity; data curation; predictive models

\section{Introduction}

The toxicity of single nanomaterials has been intensively tested in recent decades. Combinations of single nanomaterials and chemicals form nano-mixtures, which might cause co-exposure and mixture effects in living organisms. Because nanomaterials present different physicochemical properties in comparison with the properties of bulk chemicals, the mixture effects of nano-mixtures might deviate from the mixture effects of chemical mixtures (e.g., organic mixtures, metal mixtures, etc.). The in silico approach provides predictive models for reducing the experimental costs of in vitro/in vivo toxicity testing [1]. Experimental studies on the toxic effects of mixtures of different compounds have continuously increased, and conventional models, such as concentration addition (CA) and independent action (IA) models, have been frequently used to estimate the toxicity of mixtures based on the additive toxicity of single compounds [2-4]. Additionally, some quantitative structure-activity relationship (QSAR)-based models have been applied to mainly predict the toxicity of organic mixtures based on the structures of single compounds [5-9]. However, there have been few studies concerning the predictability of nano-mixture toxicity, and most of these studies employed the CA and IA models to test the applicability of the two conventional models [10-18]. The number of studies on QSARs for predicting nano-mixture toxicity is considerably lower, and thus far, there have been only three of them [10-12].

For chemical mixtures, previous reviews provided comprehensive knowledge on (1) computational models for chemical mixture toxicity $[19,20]$ and (2) underlying mechanisms of mixture effects [21]. In order to apply in silico methods to nano-mixture toxicity, datasets, models, and toxicity mechanisms would be required. Current reviews for nanomixture toxicity [22] focused only on a toxicity mechanism known as the Trojan horse phe- 
nomenon. There is a gap in the datasets and predictive models, which might cause obstacles in the toxicity assessment of nano-mixtures by using the in silico approach. To provide readers with a picture of the currently available datasets and predictive models of nanomixture toxicity, in this review, we collected and curated 183 studies [10,11,13-18,23-197] of nano-mixture toxicity to investigate the current data and model availability and gap, and we derived research challenges to facilitate further experimental studies for data gap filling and the development of predictive models.

\section{Literature Collection}

Literature was searched from Web of Science (webofknowledge.com) and Google Scholar (scholar.google.com) using the keywords "mixture", "toxicity", and "nanomaterials". Each publication was carefully checked and analyzed to appropriately gather the toxicity test datasets of mixtures of nanomaterials and other substances. Selected publications for the purpose of this review were studies assessing the co-exposure effects of nano-mixtures on in vitro models and environmental species such as crustaceans, fish, bacteria, plants, algae, and mollusks. The last search was conducted in June 2020. A total of 183 publications from 2005 to 2020 were sorted after the searching and checking process. Information on (1) nano-mixture composition, (2) toxicity tests, (3) toxicity endpoints, and (4) mixture toxicity models was extracted from the literature. Nano-mixture composition included the nanomaterials, mixed chemicals, and type of nano-mixture. Toxicity tests included the name and group of tested species and toxicity test guidelines. Toxicity endpoints are the names of the endpoints measured in the toxicity tests. Mixture toxicity models are the available models used to estimate or predict the toxicity of nano-mixtures.

\section{Current Available Predictive Models for Nano-Mixture Toxicity}

Conventional models for the joint toxicity of organic compounds include CA [2] and IA [4] models. Both models describe the additive joint toxicity of chemicals based on the similarity or dissimilarity of their mode of action (MoA). The models require the toxicity of single substances in a mixture to estimate the toxicity of the mixture as follows:

$$
\text { CA model : } \mathrm{ECx}_{\operatorname{mix}}=\sum_{1}^{n}\left(\frac{p_{i}}{\mathrm{ECx}_{i}}\right)^{-1}
$$

where $\mathrm{ECx}_{\text {mix }}$ is the predicted toxic effect of the mixture, $p_{i}$ is the fraction of component $i$ in the mixture, and $\mathrm{ECx}_{i}$ is the individual effect concentration when applied singly.

$$
\text { IA model : } \mathrm{Y}=\mathrm{u}_{\max } \prod_{1}^{n} q_{i}\left(C_{i}\right)
$$

where $\mathrm{Y}$ denotes the biological response, $C_{i}$ is the concentration of chemical $i$ in the mixture, $q_{i}\left(C_{i}\right)$ is the probability of non-response, $\mathrm{u}_{\max }$ is the control response for endpoints, and $\Pi$ is the multiplication function. Several studies applied CA and IA models to predict the toxicity of nano-mixtures and showed good predictivity (correlation coefficient value of up to 0.91) and explain the synergism and antagonism effects of the mixtures [13-18]. For example, Lopes and colleagues [13] utilized CA and IA models for mixtures of $\mathrm{ZnO} / \mathrm{Ag}$ nanoparticles (NPs) and their metal ion counterparts and showed that both Zn NP and Ag NP mixture showed a deviation from additivity and they showed synergism when the concentration of Ag ions increased and antagonism when the concentration of AgNPs increased in the suspension. The study by Wang and colleagues [15] demonstrated that toxicity predictions of CA/IA models were close to the observed joint toxicities of binary mixtures of graphene and ionic liquids. Although CA/IA models are able to provide hints about the synergistic/antagonistic effects of mixtures, experiments to determine the toxicity of all single components are needed in order to apply CA/IA models. Such a process requires high cost and time, and such experiments might not always be feasible.

QSAR models might be a helpful approach to compensate for the disadvantages of CA and IA models. QSAR models are mathematical relationships between endpoints 
(e.g., mortality, mitochondrial activity) and descriptors (e.g., concentration of mixtures, physicochemical properties of NPs). The QSAR model inputs do not require the toxicity of all single components in mixtures. Although there were 183 studies that investigated the toxicity of nano-mixtures, only three studies [10-12] developed QSAR models for the photocatalytic activity and toxicity of $\mathrm{TiO}_{2} \mathrm{NP}$-based nano-mixtures (Table 1 ). These studies were conducted to develop models for predicting the photocatalytic activity and cytotoxicity of nano-mixtures consisting of $\mathrm{TiO}_{2} \mathrm{NPs}$ and (poly) metallic clusters ( $\mathrm{Au}, \mathrm{Ag}$, $\mathrm{Pd}$, and $\mathrm{Pt}$ ). The nano-mixtures were heterogeneous photocatalytic NPs that could be used for ultraviolet and visible (UV-vis) light-induced processes to remove harmful pollutants from gas and aqueous phases. The two endpoints in these studies were the photocatalytic activity under UV-vis light of $\mathrm{TiO}_{2}$ nano-mixtures and the viability of Chinese hamster ovary (CHO-K1) cells exposed to $\mathrm{TiO}_{2}$ nano-mixtures. Descriptors of nano-mixtures $\left(D_{\text {mix }}\right)$ were calculated from quantum chemical descriptors of metallic clusters by using the additive equation:

$$
D_{\text {mix }}=\% \mathrm{~mol}_{M e_{1}} \times D_{1}+\ldots+\% \mathrm{~mol}_{M e_{n}} \times D_{n}
$$

where $D_{m i x}$ is the additive descriptor of nano-mixtures, and $\% m o l_{M e_{n}}$ and $D_{n}$ are the mole fraction and quantum chemical descriptors of metal cluster $n$ in the mixtures. The additive equation was based on a simple additive approach for joint toxicity, where the properties of heterogeneous NPs are the result of the additive contribution of each component. Two algorithms were used to develop QSAR models: multiple linear regression (MLR) and decision tree (DT). These QSAR models showed good predictive power and successfully explained the observed photocatalytic activity and cytotoxicity of heterogeneous $\mathrm{TiO}_{2} \mathrm{NPs}$ (over 90\%). However, the data for model development were limited to 29 data rows of different metal cluster fractions so the applicability domain of these models was limited to only nano-mixtures of $\mathrm{TiO}_{2} \mathrm{NPs}$ and four metal clusters (i.e., $\mathrm{Ag}, \mathrm{Au}, \mathrm{Pt}$, and $\mathrm{Pd}$ ). Furthermore, nano-toxicology regulation and assessment could adopt additional QSAR models of the toxicity of other NP-based nano-mixtures. 
Table 1. List of previous QSAR models for nano-mixtures.

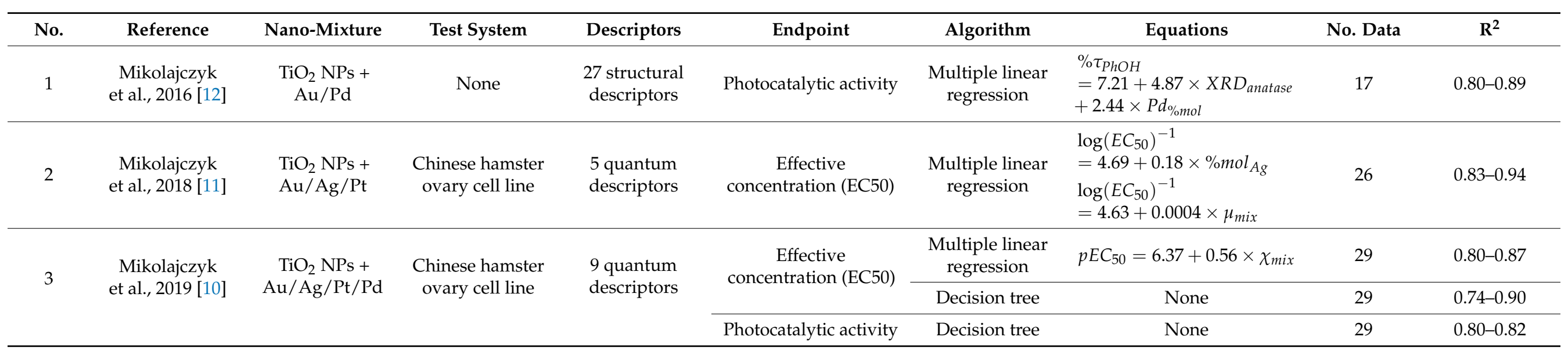


It is feasible to develop QSAR models which have a larger applicability domain for nano-mixture toxicity if larger datasets containing a variety of mixed chemicals are acquired. Our curated literature data contain studies of the in vivo/in vitro toxicity of $\mathrm{NP}-\mathrm{NP}, \mathrm{NP}-$-inorganic chemical, and NP-organic chemical mixtures (Figure $1 \mathrm{~A}, \mathrm{~B}) . \mathrm{TiO}_{2}$, fullerenes $\left(\mathrm{C}_{60}\right)$, multiwall carbon nanotubes (MWCNT), $\mathrm{ZnO}$, and $\mathrm{Ag}$ are the most popular NPs in the tested nano-mixtures (Figure 1C). The curated data have been successfully exploited to develop QSAR models for single nanomaterial toxicity by using regression and classification algorithms [198-202]. QSAR model development for nano-mixture toxicity might apply similar data curation (e.g., data collection, data gap filling, normalization, etc.) and modeling methods (algorithms, validation) as in the case of single nanomaterials. However, descriptors for nano-mixtures $\left(D_{m i x}\right)$ are very important and they are different from the descriptors of single nanomaterials. Because nano-mixtures consist of more than two components, their descriptors should contain information about all components (i.e., concentration, properties). Previous research suggested the calculation of $D_{\text {mix }}$ from the properties of all mixture components by using several methods $[5,7,8,10-12]$. The most used method is based on the assumption that components in a mixture act jointly by simple addition (Equation (3)). In addition to the additive $\mathrm{D}_{\text {mix }}$, other equations such as mean square and mean cubic $[5,7,8,10-12]$ were previously applied to calculate $D_{\text {mix }}$ of organic mixtures, and models based on these mixture descriptors showed good prediction performance $\left(R^{2}=0.71-0.94\right)$. These equations and models have not been applied to nano-mixtures yet. Although there are several available methods for calculating $D_{\text {mix }}$, they might not cover all nano-mixture toxicity data because the assumption of joint properties (e.g., additive) might not be suitable for all nano-mixtures. New methods for calculating $D_{\text {mix }}$ might be proposed during the development of QSAR models for nano-mixture toxicity. Because NPs and mixed chemicals are often different, in that one component is nano and the other(s) is bulk material, it is difficult to measure their common properties for $D_{\text {mix }}$ calculation. Instead of using experimental properties, theoretical properties are commonly used for nano-mixtures $[10-12,203,204]$. Simulation-based methods for theoretical properties can be classified as quantum mechanics (QM) or molecular dynamics (MD). Mikolajczyk and colleagues [10-12] applied QM simulation and density functional theory (DFT) calculation to small metal clusters $\left(0.5 \times 0.5 \times 0.5 \mathrm{~nm}^{3}\right)$ to obtain QM descriptors for $D_{\text {mix }}$ of heterogeneous $\mathrm{TiO}_{2} \mathrm{NPs}$. The $D_{\text {mix }}$ obtained from this $\mathrm{QM}$ approach (electronegativity) worked well to produce QSAR models that explained over $90 \%$ the observed photocatalytic activity and cytotoxicity of heterogeneous $\mathrm{TiO}_{2} \mathrm{NPs}$. Although the QM calculations could provide information on electronic energy (e.g., ionization, electronegativity, highest occupied/lowest unoccupied molecular orbital, etc.), calculations of large nano-sized systems are difficult and time-consuming, so this approach is limited by the size of NP clusters. The MD approach was utilized in the work of Burk and colleagues [203,204]. This approach simulated large Fe-Doped ZnO NPs (8-40 nm diameter) and $D_{\text {mix }}$ descriptors were based on a force-field calculation of the potential energies of whole NPs and the core and shell layers. The $D_{m i x}$ descriptors obtained from this approach showed potential for linear regression (i.e., predicting cell viability, membrane damage, and mitochondrial reactive oxygen species (ROS) of HeLa and KLN205 cells with $R^{2}=0.740-0.877$ ) and classification models (i.e., clustering toxicity data of Fe-Doped $\mathrm{ZnO}$ NPs by using principal component analysis with good accordance with the algal growth inhibition data) [204]. The MD method has a disadvantage in that it provides only topological and potential energy descriptors, which are not related to the electronic properties of nano-mixtures. In addition to the QM and MD approaches, the molecular descriptor approach might be applicable to nano-mixture toxicity data. The molecular descriptor method was previously used to obtain $D_{\text {mix }}$ for antibiotics and pesticide mixtures [204]. The QSAR models for antibiotics and pesticide mixture toxicity demonstrated high predictive performance $\left(R^{2}=0.9366\right)$. Their limitation is similar to the QM approach, namely that calculations for large-sized nano-mixtures are difficult and time-consuming. For QSAR model development of the current curated 
nano-mixture toxicity data, we suggest considering all three methods for finding the $D_{\text {mix }}$ with the best performance models and the most suitable toxicity mechanisms.

(A)

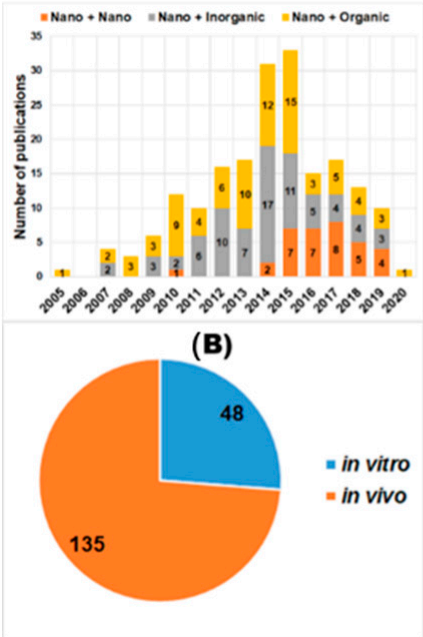

(C)

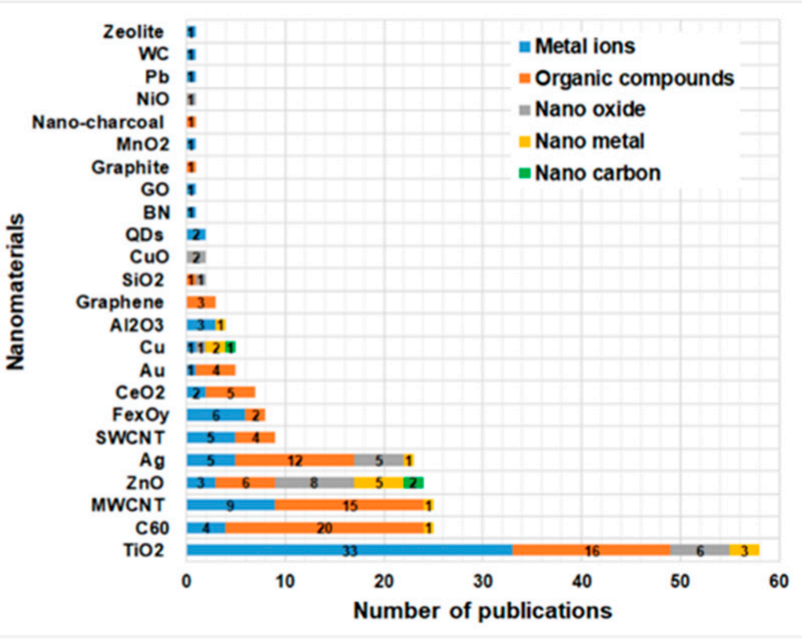

Figure 1. Number of studies on toxicity of nano-mixtures classified by year and type of nano-mixture (A), by type of toxicity test (B), and by nanomaterials and mixture components (C).

There are several algorithms for QSAR models suggested by the Organization for Economic Co-operation and Development (OECD) [205]: linear and nonlinear algorithms. For conventional organic mixtures, linear algorithms such as MLR are often used for developing models [5-9] because of their transparency and mechanistic interpretation. For nanomixtures, only MLR and DT algorithms have been used for developing models [10-12]. Nonlinear algorithms such as random forest (RF) and artificial neural network (ANN) might be useful for developing models due to their high accuracy and robustness. However, transparency and mechanistic interpretation of these algorithms are lower than MLR and DT algorithms because they do not provide equations or single trees of prediction for other users who could use direct predictions (e.g., equations, etc.). The choice of algorithm is based on the model's performance, transparency, and mechanistic interpretation. Therefore, it is time-consuming to choose the most suitable algorithms for QSARs of nano-mixture toxicity.

As a part of in silico research, nano-mixture toxicity QSAR models would provide low-cost toxicity screening of nano-mixtures, which would help to reduce animal testing and chemical waste [206]. Additionally, the models would provide warnings about potential nano-mixture toxicity when we design new nanomaterials (e.g., photocatalytic nanomaterials [10]) for safe-by-design approaches and minimal harmful effects. Another application of the models is checking the biocompatibility of new NP-based drug delivery. The NP-based drug might undergo many co-exposures to other organic chemicals and present mixture toxicity (e.g., AuNPs in presence of Polysorbate 20 synergistic toxicity at concentrations where the individual components were benign [52]). Finally, the models might help to control the processes of the release and emission of NPs into the environment, where flows of NP release might interact with dissolved organic matter and provoke mixture toxicity to the environment.

\section{Current Available Data of Nano-Mixture Toxicity}

Based on the types of mixed chemicals, nano-mixture toxicity data from 183 publications were divided into three groups: (1) NPs and NPs; (2) NPs and inorganic chemicals; and (3) NPs and organic chemicals (Figure 1A). The toxicity data were categorized into two groups: in vitro and in vivo (Figure 1B). The in vitro toxicity group included studies using bacteria, cells from animals, and humans as test systems (48 studies). The in vivo toxicity group included studies using animals as test systems (135 studies) (Figure 1B). The 
five most popular NPs in the tested nano-mixtures (i.e., $\mathrm{TiO}_{2}, \mathrm{C}_{60}, \mathrm{MWCNT}, \mathrm{ZnO}$, and $\mathrm{Ag}$ ) occupied around $70 \%$ of the curated data (Figure 1C). Their data would be helpful for the meta-analysis of the toxicity of each NP-based nano-mixture.

We categorized the tested species into nine groups: crustaceans, fish, bacteria, plants, algae, cultured cell lines, mollusks, insects and worms, and fungi and others (Figure 2A). Among these nine groups, crustaceans, fish, and bacteria were the three most popular tested species, and 111/183 studies used these species to test the toxicity of nano-mixtures (Figure 2A). Metal oxide NPs were most frequently tested on these test systems, and 95/183 studies investigated the toxicity of metal oxide-based nano-mixtures (Figure 2A). Carbonaceous nanomaterial-based nano-mixtures were the second largest group, where 60/183 studies investigated their toxicity (Figure 2A). We further categorized 111 studies of crustaceans, fish, and bacteria into smaller groups of nanomaterials and species (Figure 2B-D). In the crustacean group, Daphnia magna (D. magna) was the most tested species, with 38/48 studies (Figure 2B). In the fish group, Danio rerio (D. rerio) was the most popular species, tested in 17/43 studies (Figure 2C). In the bacterial group, Escherichia coli (E. coli) was the most tested species, with $8 / 20$ studies. $\mathrm{TiO}_{2}$ based nano-mixtures were most commonly tested for toxicity to crustaceans (13/48 studies) and fish (17/43 studies) (Figure $2 \mathrm{~B}, \mathrm{C}$ ). $\mathrm{C}_{60}$-based nano-mixtures were the second largest nano-mixture group that was tested for toxicity to crustaceans (10/48 studies) and fish (5/43 studies) (Figure 2B,C). In the bacterial group, Ag NPs and ZnO NP-based nano-mixtures were the largest groups (10/20 studies).
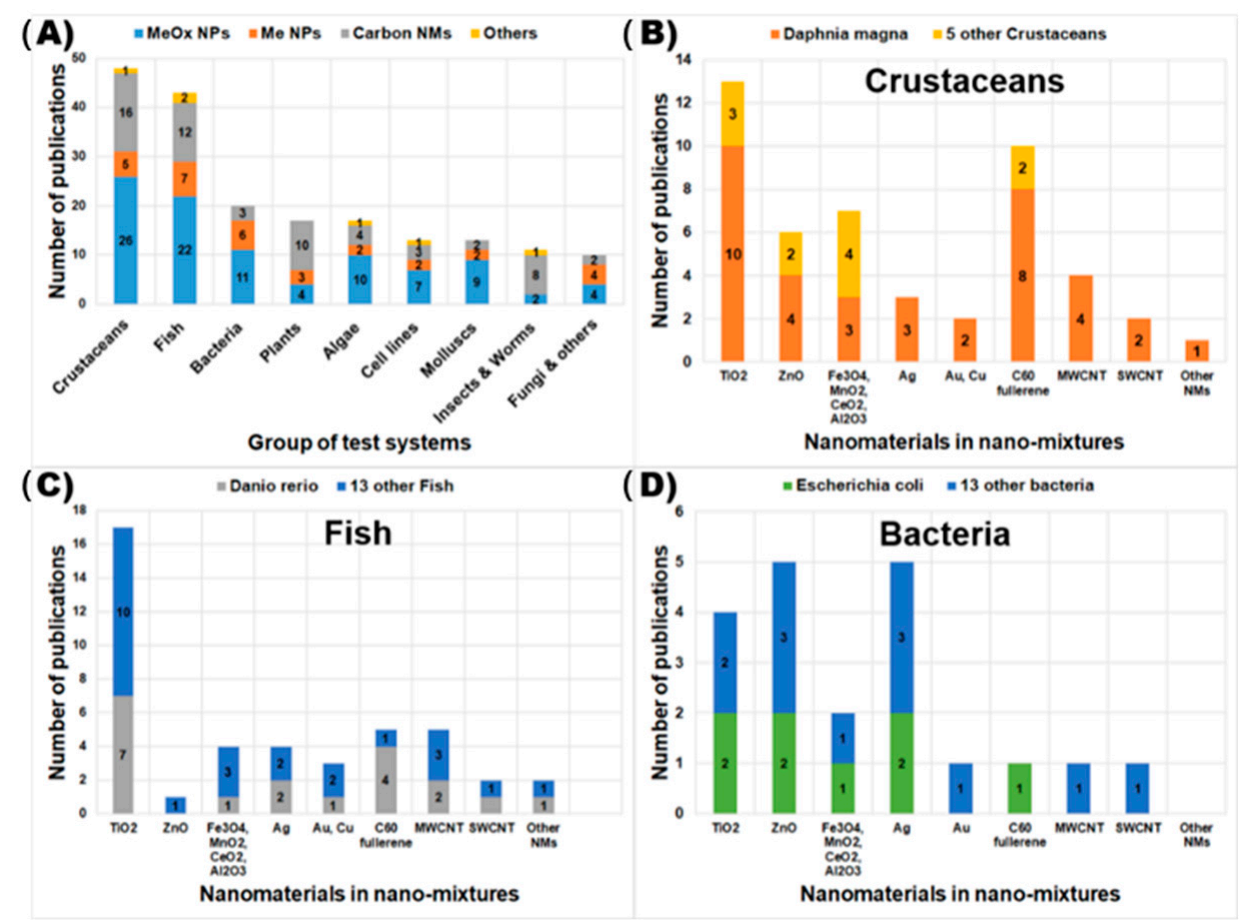

Figure 2. Number of studies on toxicity of nano-mixtures classified by group of test systems (A). Number of studies on toxicity of nano-mixtures classified by nanomaterials in nano-mixtures for crustaceans (B), fish (C), and bacteria (D). (SWCNT: single-walled carbon nanotube, MWCNT: multiple-walled carbon nanotube, NMs: nanomaterials).

Among the 183 studies, there were 88 different species and 48 different toxicity endpoints for all tested species. The endpoints of the three most popular species (D. magna, D. rerio, and E. coli) are shown in Figure 3. For D. magna, mortality and immobilization were the most abundant endpoints ( $45 \%$ ), followed by bioaccumulation $(23 \%)$, reproduction $(8 \%)$, and oxidative stress $(7 \%)$. Other toxicity endpoints $(17 \%)$ for $D$. magna were uptake, metal ATPase activity, metallothionein inhibition, retention of dietary, cell damage, and 
hatching rate. For D. rerio, mortality was the most measured endpoint (19\%), followed by bioaccumulation $(17 \%)$, gene expression $(15 \%)$, oxidative stress $(10 \%)$, and hatching rate $(8 \%)$. Other endpoints ( $31 \%$ ) for D. rerio were cell viability, locomotion activity, malformation, abnormality rate, glutathione level, heartbeat, mitochondrial activity, thyroid hormone content, and uptake. For E. coli, growth inhibition was the most measured endpoint (40\%). Other endpoints $(60 \%)$ were oxidative stress, ATP production, photoproduction, indigo degradation, cell wall damage, cell viability, and bioaccumulation.

(A)

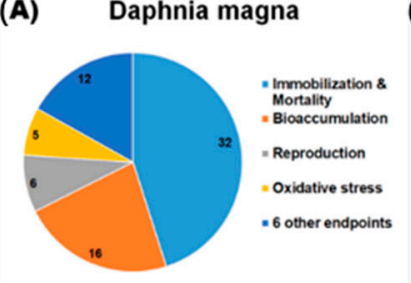

(B) Danio rerio

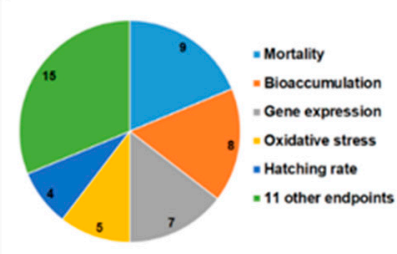

(C) Escherichia coll

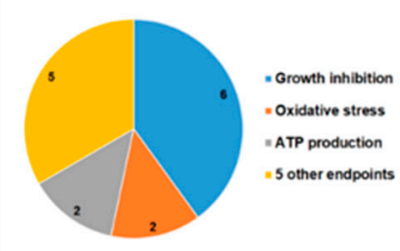

Figure 3. Number of studies on toxicity of nano-mixtures classified by endpoints for D. magna (A), D. rerio (B), and E. coli (C).

$\mathrm{TiO}_{2} \mathrm{NPs}$ are often mixed with metal ions such as $\mathrm{Cd}^{2+}, \mathrm{Cu}^{2+}$, and $\mathrm{As}^{5+}$ and organic compounds such as pesticides and antibiotics for toxicity testing. $\mathrm{C}_{60}$ and MWCNTs are often mixed with organic compounds such as pesticides and antibiotics for toxicity testing. $\mathrm{ZnO}$ NPs are often mixed with nanometals/oxides and organic compounds for toxicity testing (Figure 1C).

In the 183 collected studies, nano-mixture properties were described by the properties of NPs and mixture components including core diameter, length (for carbon nanotubes), hydrodynamic diameter, surface charge, surface area, chemical composition, and crystallinity. If the mixture components were nanomaterials, then the core diameter was usually described. For inorganic/organic compounds, chemical names or formulas were provided.

As mentioned in the previous sections, the major test systems are D. magna, D. rerio, and E. coli. We summarize the data of these three species in Tables 2-4. Among the various nanomaterials tested for these species, $\mathrm{TiO}_{2} \mathrm{NPs}, \mathrm{ZnO} \mathrm{NPs}$, and $\mathrm{C}_{60}$ fullerenes were the most tested nanomaterials (Figure 2). According to OECD guidelines [205], to develop reliable QSAR models, defined endpoints should be considered. As shown in Figure 3, there are 34 endpoints for D. magna, D. rerio, and E. coli. 
Table 2. Summary of current nano-mixture toxicity data for D. magna (CA: Concentration Addition, IA: Independent Action).

\begin{tabular}{|c|c|c|c|c|c|c|c|}
\hline No. & Article & Nanomaterials & Mixed Substance & $\begin{array}{c}\text { Mixed Substance } \\
\text { Type }\end{array}$ & $\begin{array}{c}\text { Toxicity Test } \\
\text { Guideline }\end{array}$ & Toxicity Endpoint & $\begin{array}{c}\text { Mixture Toxicity } \\
\text { Models }\end{array}$ \\
\hline 1 & $\begin{array}{l}\text { Azevedo et al., } 2017 \text { Science of } \\
\text { the Total Environment [14] }\end{array}$ & $\mathrm{ZnO}$ NPs & Ag NPs & Nanometal & OECD 202; OECD 211 & $\begin{array}{l}\text { Immobilization, } \\
\text { Reproduction }\end{array}$ & CA and IA \\
\hline 2 & $\begin{array}{c}\text { Baun et al., } 2008 \\
\text { Aquatic Toxicology [98] }\end{array}$ & C60 fullerenes & $\begin{array}{c}\text { Atrazine, } \\
\text { Methylparathione, } \\
\text { Pentachlorophenol, } \\
\text { Phenanthrene }\end{array}$ & Organic compounds & OECD 211, ISO 6341 & $\begin{array}{l}\text { Reproduction, } \\
\text { Immobilization, } \\
\text { Bioaccumulation }\end{array}$ & None \\
\hline 4 & $\begin{array}{c}\text { Cano et al., } 2018 \\
\text { Environmental Science and } \\
\text { Technology [178] }\end{array}$ & MWCNT & $\mathrm{Cu}^{2+}$ & Metal ions & None & $\begin{array}{l}\text { Metallothionein } \\
\text { inhibition, } \\
\text { Bioaccumulation }\end{array}$ & None \\
\hline 5 & $\begin{array}{l}\text { Fan et al., } 2011 \\
\text { Environmental Pollution [107] }\end{array}$ & $\mathrm{TiO}_{2} \mathrm{NPs}$ & $\mathrm{Cu}^{2+}$ & Metal ions & None & $\begin{array}{c}\text { Metallothionein } \\
\text { inhibition, Mortality, } \\
\text { Bioaccumulation }\end{array}$ & None \\
\hline 7 & $\begin{array}{c}\text { Fang et al., } 2011 \\
\text { Environmental Toxicology and } \\
\text { Chemistry [153] }\end{array}$ & Nano-charcoal & Tributyltin, Dibutyltin & Organometallic & ISO 6341 & $\begin{array}{c}\text { Reproduction, } \\
\text { Immobilization, } \\
\text { Bioaccumulation }\end{array}$ & None \\
\hline 8 & $\begin{array}{c}\text { Gao et al., } 2018 \text { Ecotoxicology } \\
\text { and Environmental Safety } \\
{[181]}\end{array}$ & $\begin{array}{l}\mathrm{MnO}_{2} \mathrm{NPs}, \mathrm{Nano}^{-} \\
\text {hydroxyapatite }\end{array}$ & $\mathrm{Cd}^{2+}$ & Metal ions & OECD 202 & $\begin{array}{l}\text { Immobilization, } \\
\text { Oxidative stress }\end{array}$ & None \\
\hline 9 & $\begin{array}{c}\text { Han et al., } 2012 \\
\text { Chinese Journal of } \\
\text { Geochemistry [158] }\end{array}$ & $\mathrm{CeO}_{2} \mathrm{NPs}$ & Atrazine & Organic compounds & US. EPA 2001 & $\begin{array}{l}\text { Reproduction, } \\
\text { Mortality, } \\
\text { Bioaccumulation }\end{array}$ & None \\
\hline 10 & $\begin{array}{l}\text { Hartmann et al., } 2012 \\
\text { Aquatic Toxicology [114] }\end{array}$ & $\mathrm{TiO}_{2} \mathrm{NPs}$ & $\mathrm{Cu}^{2+}$ & Metal ions & OECD 202, ISO 6341 & Bioaccumulation & None \\
\hline
\end{tabular}


Table 2. Cont

\begin{tabular}{|c|c|c|c|c|c|c|c|}
\hline No. & Article & Nanomaterials & Mixed Substance & $\begin{array}{c}\text { Mixed Substance } \\
\text { Type }\end{array}$ & $\begin{array}{l}\text { Toxicity Test } \\
\text { Guideline }\end{array}$ & Toxicity Endpoint & $\begin{array}{c}\text { Mixture Toxicity } \\
\text { Models }\end{array}$ \\
\hline 11 & $\begin{array}{l}\text { Kim et al., } 2009 \text { Environmental } \\
\text { Science \& Technology [136] }\end{array}$ & MWCNT & $\mathrm{Cu}^{2+}$ & Metal ions & None & $\begin{array}{l}\text { Bioaccumulation, } \\
\text { Mortality, Oxidative } \\
\text { stress }\end{array}$ & None \\
\hline 12 & $\begin{array}{l}\text { Kim et al., } 2010 \text { Environmental } \\
\text { Toxicology and Chemistry [73] }\end{array}$ & $\mathrm{Cu}$ NPs & SWCNT & Nanocarbon & None & $\begin{array}{c}\text { Mortality, } \\
\text { Bioaccumulation }\end{array}$ & None \\
\hline 13 & $\begin{array}{c}\text { Lopes et al., } 2016 \\
\text { J. Hazardous Materials [13] }\end{array}$ & Ag NPs, ZnO NPs & $\begin{array}{l}\text { Ag NPs, } \mathrm{ZnO} \mathrm{NPs,} \\
\mathrm{Ag}^{+}, \mathrm{Zn}^{2+}\end{array}$ & $\begin{array}{l}\text { Nano-oxide, Metal } \\
\text { ions }\end{array}$ & OECD 202 & Immobilization & CA and IA \\
\hline 14 & $\begin{array}{l}\text { Martín-de-Lucía et al., } 2019 \\
\text { Science of The Total } \\
\text { Environment [16] }\end{array}$ & Graphite-diamond & $\begin{array}{l}\text { Fungicide } \\
\text { thiabendazole }\end{array}$ & Organic compounds & OECD 202 & Immobilization & CA and IA \\
\hline 15 & $\begin{array}{l}\text { Molins-Delgado et al., } 2016 \\
\text { Environmental Research [184] }\end{array}$ & Ag NPs & $\begin{array}{c}\text { Benzophenone, } \\
\text { Ethyl-PABA, } \\
\text { 4-methylbenzylidene } \\
\text { camphor, } \\
\text { Ethylhexyl-methoxy } \\
\text { cinamate }\end{array}$ & Organic compounds & ISO 6341 & Immobilization & None \\
\hline 16 & $\begin{array}{c}\text { Park et al., } 2019 \\
\text { Journal of Nanoparticle } \\
\text { Research [117] }\end{array}$ & $\mathrm{TiO}_{2}$ NPs, ZnO NPs & $\mathrm{Ag}+$ & Metal ions & OECD 202 & Immobilization & None \\
\hline 17 & $\begin{array}{l}\text { Park et al., } 2019 \\
\text { Molecular \& Cellular } \\
\text { Toxicology [81] }\end{array}$ & $\mathrm{Fe}_{3} \mathrm{O}_{4} \mathrm{NPs}$ & $\mathrm{Zn}^{2+}$ & Metal ions & OECD 202 & Immobilization & CA and IA \\
\hline 18 & $\begin{array}{l}\text { Rosenfeldt et al., } 2014 \\
\text { Environmental Science \& } \\
\text { Technology [112] }\end{array}$ & $\mathrm{TiO}_{2} \mathrm{NPs}$ & $\mathrm{Cu}^{2+}, \mathrm{Ag}^{+}, \mathrm{As}^{5+}$ & Metal ions & OECD 202 & $\begin{array}{l}\text { Bioaccumulation, } \\
\text { Immobilization }\end{array}$ & None \\
\hline 19 & $\begin{array}{c}\text { Rosenfeldt et al., } 2015 \\
\text { Environmental Science \& } \\
\text { Technology [113] }\end{array}$ & $\mathrm{TiO}_{2}$ NPs & $\mathrm{Cu}^{2+}$ & Metal ions & OECD 202 & $\begin{array}{l}\text { Bioaccumulation, } \\
\text { Immobilization }\end{array}$ & None \\
\hline 20 & $\begin{array}{c}\text { Sanchis et al., } 2016 \\
\text { Environmental Science \& } \\
\text { Technology [59] }\end{array}$ & C60 fullerenes & $\begin{array}{l}\text { Nonylphenol, } \\
\text { Triclosan, Malathion, } \\
\text { Diuron, Glyphosate }\end{array}$ & Organic compounds & OECD 202, ISO 6341 & Immobilization & None \\
\hline
\end{tabular}


Table 2. Cont.

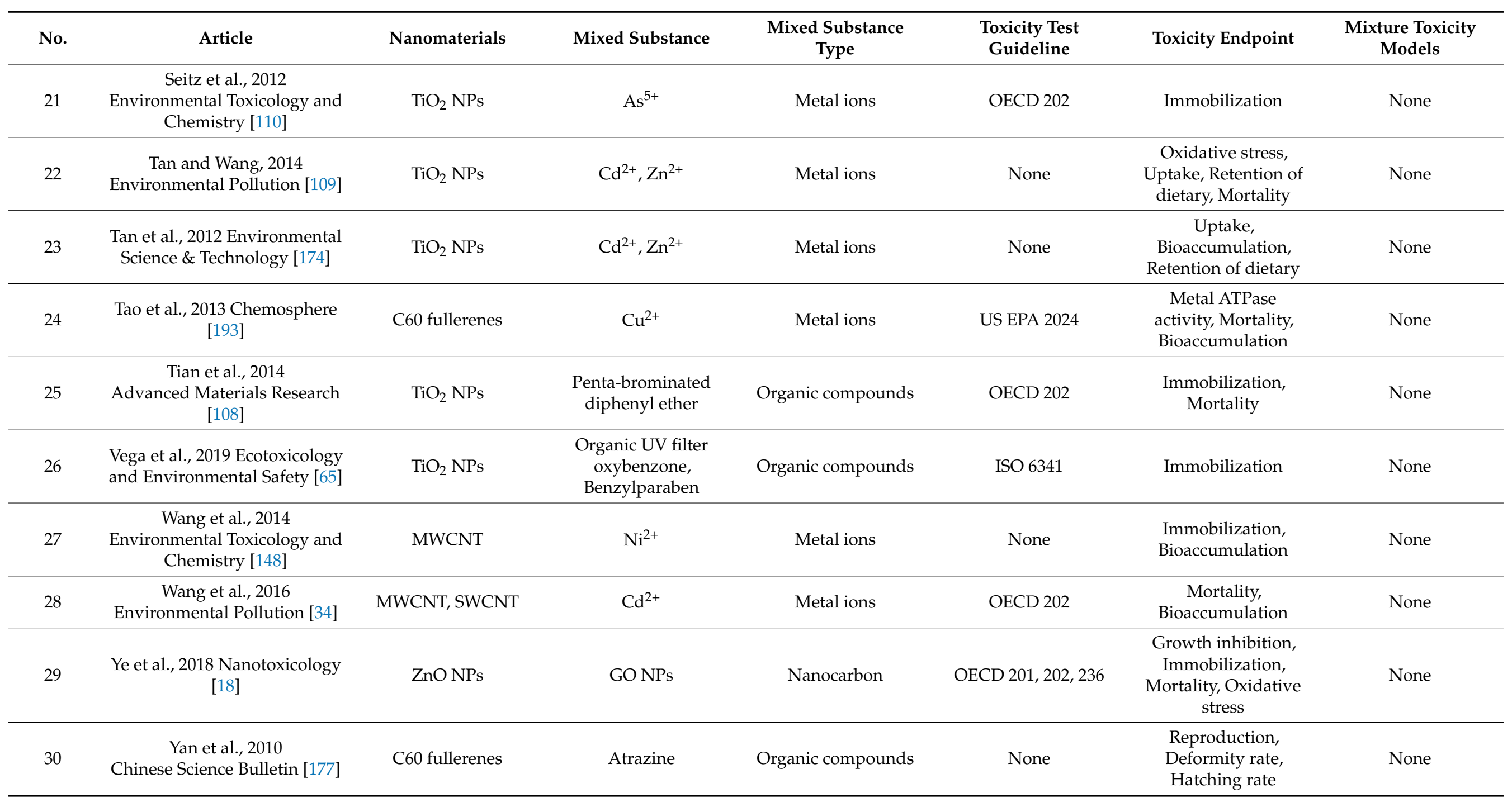


Table 2. Cont

\begin{tabular}{|c|c|c|c|c|c|c|c|}
\hline No. & Article & Nanomaterials & Mixed Substance & $\begin{array}{c}\text { Mixed Substance } \\
\text { Type }\end{array}$ & $\begin{array}{c}\text { Toxicity Test } \\
\text { Guideline }\end{array}$ & Toxicity Endpoint & $\begin{array}{c}\text { Mixture Toxicity } \\
\text { Models }\end{array}$ \\
\hline 31 & $\begin{array}{c}\text { Yang et al., } 2010 \\
\text { Aquatic Toxicology [189] }\end{array}$ & C60 fullerenes & Fluoranthene & Organic compounds & EPA 6004-90027 & $\begin{array}{c}\text { Immobilization, Cell } \\
\text { damage }\end{array}$ & None \\
\hline 32 & $\begin{array}{c}\text { Ye et al., } 2018 \text { Nanotoxicology } \\
\text { [18] }\end{array}$ & $\mathrm{ZnO} N P s$ & GO NPs & Nanocarbon & OECD 201, 202, 236 & $\begin{array}{l}\text { Growth inhibition, } \\
\text { Mortality, } \\
\text { Immobilization }\end{array}$ & None \\
\hline 33 & $\begin{array}{c}\text { Yu \& Wang, } 2013 \text { Water } \\
\text { Research [197] }\end{array}$ & MWCNT, SWCNT & $\mathrm{Cd}^{2+}, \mathrm{Zn}^{2+}$ & Metal ions & None & $\begin{array}{c}\text { Uptake, } \\
\text { Bioaccumulation }\end{array}$ & None \\
\hline 34 & $\begin{array}{c}\text { Yu \& Wang, } 2014 \\
\text { Environmental Toxicology and } \\
\text { Chemistry [26] }\end{array}$ & C60 fullerenes & $\mathrm{Cd}^{2+}, \mathrm{Zn}^{2+}$ & Metal ions & None & Bioaccumulation & None \\
\hline 35 & $\begin{array}{l}\text { Zhang et al., } 2015 \text { Journal of } \\
\text { Environmental Sciences [31] }\end{array}$ & Ag NPs & $\mathrm{Ag}^{+}$ & Metal ions & OECD 202 & Mortality & None \\
\hline
\end{tabular}

Table 3. Summary of current nano-mixture toxicity data for D. rerio.

\begin{tabular}{|c|c|c|c|c|c|c|c|}
\hline No. & Article & Nanomaterials & Mixed Substance & $\begin{array}{c}\text { Mixed Substance } \\
\text { Type }\end{array}$ & $\begin{array}{c}\text { Toxicity Test } \\
\text { Guideline }\end{array}$ & Toxicity Endpoint & $\begin{array}{c}\text { Mixture Toxicity } \\
\text { Models }\end{array}$ \\
\hline 1 & $\begin{array}{c}\text { Azevedo Costa et al., } 2012 \\
\text { Comparative Biochemistry } \\
\text { and Physiology, Part C } \\
{[176]}\end{array}$ & C60 fullerenes & $\mathrm{As}^{3+}$ & Metal ions & None & $\begin{array}{l}\text { Cell viability, Mitochondrial } \\
\text { dehydrogenase functionality, } \\
\text { Oxidative stress, } \\
\text { Bioaccumulation }\end{array}$ & None \\
\hline 2 & $\begin{array}{l}\text { Fang et al., } 2015 \text { Journal of } \\
\text { Hazardous Materials [160] }\end{array}$ & $\mathrm{TiO}_{2} \mathrm{NPs}$ & Pentachlorophenol & $\begin{array}{l}\text { Organic } \\
\text { compounds }\end{array}$ & None & $\begin{array}{c}\text { Bioaccumulation, Oxidative } \\
\text { stress, Gene expression, } \\
\text { Glutathione level }\end{array}$ & None \\
\hline 3 & $\begin{array}{c}\text { Ferreira et al., } 2014 \\
\text { Aquatic Toxicology [131] }\end{array}$ & C60 fullerenes & Benzo[a]pyrene & $\begin{array}{c}\text { Organic } \\
\text { compounds }\end{array}$ & None & $\begin{array}{l}\text { Cell viability, Oxidative } \\
\text { stress, Bioaccumulation, } \\
\text { Glutathione level }\end{array}$ & None \\
\hline
\end{tabular}


Table 3. Cont.

\begin{tabular}{|c|c|c|c|c|c|c|c|}
\hline No. & Article & Nanomaterials & Mixed Substance & $\begin{array}{c}\text { Mixed Substance } \\
\text { Type }\end{array}$ & $\begin{array}{c}\text { Toxicity Test } \\
\text { Guideline }\end{array}$ & Toxicity Endpoint & $\begin{array}{c}\text { Mixture Toxicity } \\
\text { Models }\end{array}$ \\
\hline 4 & $\begin{array}{c}\text { Ginzburg et al., 2018 ACS } \\
\text { Nano [52] }\end{array}$ & $\mathrm{Au}$ NPs & Polysorbate 20 & $\begin{array}{c}\text { Organic } \\
\text { compounds }\end{array}$ & None & Mortality & None \\
\hline 5 & $\begin{array}{c}\text { Henry et al., } 2007 \\
\text { Environmental Health } \\
\text { Perspectives [56] }\end{array}$ & C60 fullerenes & Tetrahydrofuran & $\begin{array}{l}\text { Organic } \\
\text { compounds }\end{array}$ & None & Mortality, Gene expression & None \\
\hline 6 & $\begin{array}{c}\text { Henry et al., } 2013 \\
\text { Environmental Science \& } \\
\text { Technology [36] }\end{array}$ & C60 fullerenes & $\mathrm{Hg}+$ & Metal ions & Plymouth University & $\begin{array}{l}\text { Mortality, Bioaccumulation, } \\
\text { Gene expression }\end{array}$ & None \\
\hline 7 & $\begin{array}{c}\text { Hu et al., } 2011 \\
\text { Environmental Pollution } \\
{[38]}\end{array}$ & $\mathrm{TiO}_{2}$ NPs & $\mathrm{Cd}^{2+}$ & Metal ions & None & Bioaccumulation & None \\
\hline 8 & $\begin{array}{l}\text { Hua et al., } 2016 \\
\text { NanoImpact [74] }\end{array}$ & $\mathrm{TiO}_{2}$ NPs & $\mathrm{ZnO}$ NPs & Nano-oxide & OECD 157 & Mortality & RA and CA \\
\hline 9 & $\begin{array}{l}\text { Krysanov \& Demidova, } \\
2012 \text { Doklady Biological } \\
\text { Sciences [146] }\end{array}$ & $\mathrm{CeO}_{2} \mathrm{NPs}$ & Doxorubicin & $\begin{array}{l}\text { Organic } \\
\text { compounds }\end{array}$ & None & $\begin{array}{l}\text { Malformations, Hatching } \\
\text { rate }\end{array}$ & None \\
\hline 10 & $\begin{array}{l}\text { Miao, } 2015 \text { Aquatic } \\
\text { Toxicology [119] }\end{array}$ & $\mathrm{TiO}_{2} \mathrm{NPs}$ & $\mathrm{Pb}^{2+}$ & Metal ions & None & $\begin{array}{c}\text { Gene expression, } \\
\text { Locomotion activity, Thyroid } \\
\text { hormone content, } \\
\text { Bioaccumulation }\end{array}$ & None \\
\hline 11 & $\begin{array}{c}\text { Park et al., } 2011 \\
\text { Nanotoxicolog y [190] }\end{array}$ & C60 fullerenes & $17 \alpha$-Ethinyl-estradiol & $\begin{array}{c}\text { Organic } \\
\text { compounds }\end{array}$ & None & $\begin{array}{c}\text { Bioaccumulation, Gene } \\
\text { expression }\end{array}$ & None \\
\hline 12 & $\begin{array}{l}\text { Park et al., } 2015 \text { Molecular } \\
\text { \& Cellular Toxicology [60] }\end{array}$ & Ag NPs & Ag nanotube & Nanometal & None & Gene expression & None \\
\hline 13 & $\begin{array}{l}\text { Pavagadhi et al., } 2014 \\
\text { Water Research [77] }\end{array}$ & Ag NPs, $\mathrm{TiO}_{2} \mathrm{NPs}$ & $\begin{array}{c}\mathrm{Ni}^{2+}, \mathrm{Mg}^{2+}, \mathrm{Zn}^{2+}, \\
\mathrm{Cu}^{2+}, \mathrm{Cd}^{2+}, \mathrm{Fe}^{2+}, \mathrm{Cr}^{3+}, \\
\mathrm{Hg}^{2+}, \mathrm{As}^{5+}, \mathrm{Al}^{3+} \\
\mathrm{Pb}^{2+}, \mathrm{Mn}^{2+}\end{array}$ & Metal ions & None & $\begin{array}{l}\text { Mortality, Heartbeat, } \\
\text { Hatching rate, Uptake }\end{array}$ & None \\
\hline
\end{tabular}


Table 3. Cont.

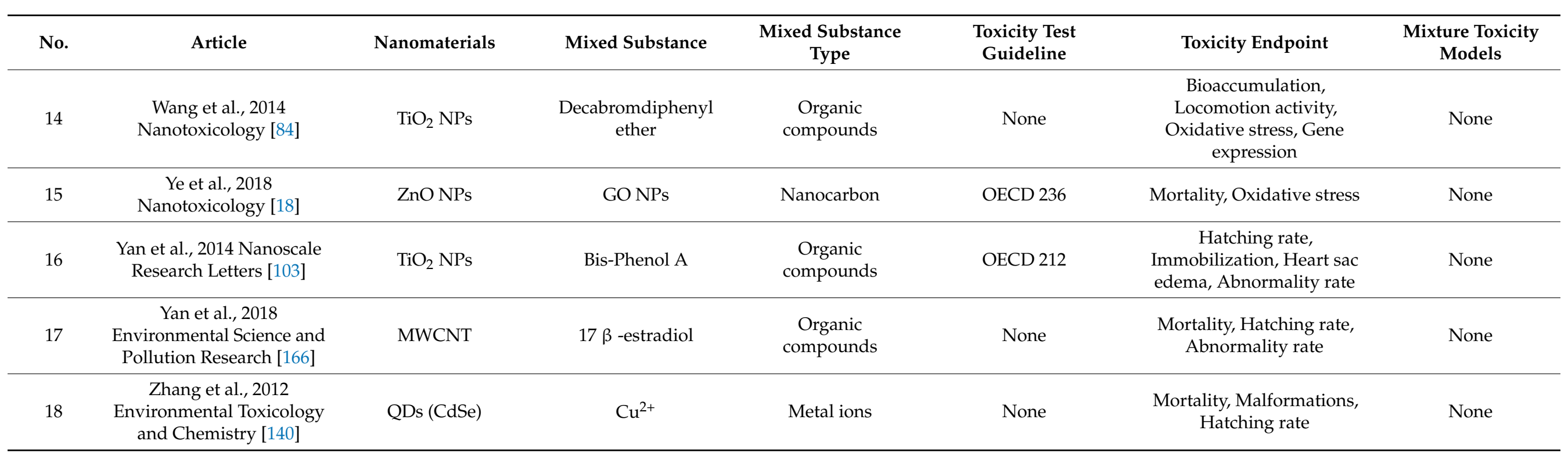


Table 4. Summary of current nano-mixture toxicity data for E. coli.

\begin{tabular}{|c|c|c|c|c|c|c|c|}
\hline No. & Article & Nanomaterials & Mixed Substance & $\begin{array}{c}\text { Mixed Substance } \\
\text { Type }\end{array}$ & $\begin{array}{c}\text { Toxicity Test } \\
\text { Guideline }\end{array}$ & Toxicity Endpoint & $\begin{array}{c}\text { Mixture Toxicity } \\
\text { Models }\end{array}$ \\
\hline 1 & $\begin{array}{l}\text { Cuahtecontzi-Delint et al., } \\
2013 \text { International Journal of } \\
\text { Chemical Reactor } \\
\text { Engineering [138] }\end{array}$ & $\mathrm{CeO}_{2} \mathrm{NPs}$ & $\begin{array}{c}\text { Surfactants (Tween 80, } \\
\text { Triton X114, and } \\
\text { Polyvinyl Pyrrolidone) }\end{array}$ & $\begin{array}{l}\text { Organic } \\
\text { compounds }\end{array}$ & None & Growth inhibition & None \\
\hline 2 & $\begin{array}{c}\text { Li et al., } 2005 \\
\text { Nanotechnology [134] }\end{array}$ & Ag NPs & Antibiotics & $\begin{array}{l}\text { Organic } \\
\text { compounds }\end{array}$ & None & Growth inhibition & None \\
\hline 4 & $\begin{array}{c}\text { Shahverdi et al., } 2007 \\
\text { Nanomedicine } \\
\text { Nanotechnology, Biology } \\
\text { and Medicine [129] }\end{array}$ & Ag NPs & Antibiotics & $\begin{array}{l}\text { Organic } \\
\text { compounds }\end{array}$ & None & Growth inhibition & None \\
\hline 5 & $\begin{array}{l}\text { Silveira et al., } 2015 \text { Journal of } \\
\text { Nanoparticle Research [147] }\end{array}$ & Ag NPs & $\begin{array}{l}\text { Hexadecylpyridinium } \\
\text { salicylate }\end{array}$ & $\begin{array}{c}\text { Organic } \\
\text { compounds }\end{array}$ & None & Growth inhibition & None \\
\hline 7 & $\begin{array}{c}\text { Tong et al., } 2015 \\
\text { Environmental Science \& } \\
\text { Technology [120] }\end{array}$ & $\mathrm{ZnO} \mathrm{NPs}$ & $\mathrm{TiO}_{2} \mathrm{NPs}$ & Nano-oxide & None & $\begin{array}{l}\text { Photoproduction, ATP } \\
\text { production, Oxidative } \\
\text { stress, Bioaccumulation }\end{array}$ & None \\
\hline 8 & $\begin{array}{c}\text { Wilke et al., } 2016 \\
\text { Environmental Science and } \\
\text { Technology [57] }\end{array}$ & $\mathrm{TiO}_{2} \mathrm{NPs}$ & Ag NPs & Nanometal & None & ATP production & None \\
\hline 9 & $\begin{array}{c}\text { Zhang et al., } 2009 \\
\text { Environmental Science \& } \\
\text { Technology [37] }\end{array}$ & C60 fullerenes & Tetrahydrofuran (THF) & $\begin{array}{l}\text { Organic } \\
\text { compounds }\end{array}$ & None & $\begin{array}{c}\text { Growth inhibition, Indigo } \\
\text { degradation }\end{array}$ & None \\
\hline
\end{tabular}


For D. magna, immobilization and mortality endpoints are defined based on two test guidelines, OECD 202 and ISO 6341, where the number of immobilized or dead D. magna is recorded and converted to a percentage over control samples. Because of their popularity in toxicity endpoints of D. magna (32 studies, $45 \%$ data), immobilization and mortality datasets might provide a potential dataset with a large applicability domain for QSAR model development. In order to develop nano-mixture QSAR models to predict immobilization/mortality, one might start with a dataset containing only one NP, such as $\mathrm{TiO}_{2}$, $\mathrm{ZnO}$, etc., to test the performance and mechanistic interpretation of models and then extend the nano-mixture dataset to other NPs and widen the applicability domain of the models. The bioaccumulation endpoint is the gradual accumulation of substances such as NPs, metal ions, or other compounds in an organism (e.g., D. magna, D. rerio, etc.). There are sixteen studies investigating the bioaccumulation of D. magna (Figure 3A) of six nanomaterials $\left(\mathrm{TiO}_{2}, \mathrm{CeO}_{2}, \mathrm{Cu}, \mathrm{MWCNT}, \mathrm{C}_{60}\right.$, and SWCNTs), six inorganic compounds $\left(\mathrm{Cu}\left(\mathrm{NO}_{3}\right)_{2}, \mathrm{CuCl}_{2}, \mathrm{CdCl}_{2}, \mathrm{ZnCl}_{2}, \mathrm{AgNO}_{3}\right.$, and $\left.\mathrm{Na}_{2} \mathrm{HAsO}_{4}\right)$, and five organic compounds (atrazine, methylparathione, pentachlorophenol, phenanthrene, and tributyltin). Previous studies suggested a mechanism by which higher bioaccumulation induced by the absorption of metal ions/dissolved organic matter and a low level of agglomeration might cause higher immobilization/mortality of D. magna $[16,59,81,117,153,184]$. For example, Martin-de-Lucia and colleagues [16] found that at low concentrations, the binary mixtures of graphite-diamond nanoparticles and fungicide thiabendazole expressed synergistic toxic interactions, which could be attributed to the increased bioavailability of fungicide thiabendazole adsorbed on graphite-diamond nanoparticles. At higher concentrations, because of agglomeration, the bioaccumulation decreased and so did the toxicity. Reproduction is another toxicity endpoint for D. magna, which follows the OECD 211 test guideline [207]. In the test guideline, the number of offspring produced by each parent animal is counted for the test and control samples. The reproduction dataset of $D$. magna might be a candidate for the development of QSAR models. Oxidative stress is the endpoint of measuring the production of reactive oxygen species (ROS). The ROS induction of nano-mixtures might be caused by the photocatalytic activity of Ag [14] or ion exchange and electrostatic adsorption to form surface complexes [181]. Data for each reactive oxygen species should be curated to obtain sufficient data to develop QSAR models.

For $D$. rerio, mortality is the most abundant endpoint in the current data collection (9 studies, 19\% data). Endpoint measurement is based on OECD 236 test guidelines [208]; therefore, it is a well-defined endpoint for QSAR model development. There are only eight studies of $D$. rerio bioaccumulation (Figure $3 \mathrm{~B}$ ) for $\mathrm{TiO}_{2}, \mathrm{C}_{60}$, and eight organic/inorganic compounds. Bioaccumulation of nano-mixtures might be proportional to their toxicity to D. rerio $[52,74,190]$ because of mixed chemicals (e.g., Polysorbate 20, etc.) assembled on the nanoparticle surfaces [52]. The hatching rate of D. rerio is an endpoint based on the OEDC 212 test guideline [209]; thus, it might be a well-defined endpoint for QSAR model development. Gene expression is a complicated endpoint due to various types of genes. Gene expression data are useful for establishing adverse outcome pathways (AOP) [210]. However, with seven studies investigating gene expression (Figure 3B) for D. rerio, welldefined toxicity endpoints relating to gene expression are important in QSAR model development and possible additional data need to be collected/produced in the future.

For E. coli, the current collected data only relate to fifteen studies including eight endpoints such as growth inhibition, ATP production, oxidative stress, and cell wall damage. The main data contain $\mathrm{TiO}_{2}, \mathrm{ZnO}$, and Ag NP-based nano-mixtures. The data might be helpful for understanding the antibacterial effects of nano-mixtures and datasets for each endpoint could be exploited in QSAR model development.

\section{Conclusions}

In this review, we collected the current 183 studies (2005-2020) for nano-mixture toxicity and described current data and predictive models for nano-mixture toxicity. We found that, in the current data on nano-mixture toxicity, the D. magna, D. rerio, and E. coli 
datasets are the three datasets containing the most studies (38, 17, and 8, respectively). We suggest additional curation of these data for the development of QSAR models with respect to toxicity endpoints. In particular, based on a total of thirty-four toxicity endpoints of these three species, three specific datasets with well-defined endpoints would be potentially useful for QSAR model development: immobilization and mortality of D. magna; mortality of D. rerio, and growth inhibition of E. coli. Data for other endpoints might need further curation and additional experimental data to develop QSAR models. We also suggest potential descriptors for QSAR model development: mixture descriptors $\left(D_{\text {mix }}\right)$ could be calculated from descriptors of single components in mixtures obtained by quantum mechanics, molecular dynamics, and molecular descriptor approaches. The available formula of $D_{\text {mix }}$ based on the assumption of joint properties in mixtures might not always be suitable for nano-mixtures so a new formula for $D_{\text {mix }}$ might be required. A variety of linear and nonlinear algorithms, such as MLR, DT, RF, and ANN, might be used for developing QSAR models. The choice of algorithm depends on the nature of the datasets, predictive performance, and possible mechanistic interpretation. Future studies that apply these datasets for QSAR models of nanomaterial toxicity will be conducted to contribute to the risk assessment of nano-mixtures.

Supplementary Materials: The table containing the list of 183 curated literature is available at: https:/ / www.mdpi.com/2079-4991/11/1/124/s1.

Author Contributions: Conceptualization, J.K., T.X.T.; data collection, T.X.T.; writing—original draft preparation, T.X.T.; writing-review and editing, T.X.T. and J.K. All authors have read and agreed to the published version of the manuscript.

Funding: This study was funded by the Korea Research Institute of Chemical Technology (KRICT) through the Development of Chemical Safety Platform Technologies (Project No. KK2052-10) and the Korea Environment Industry \& Technology Institute (KEITI) through "the Technology Program for Establishing Biocide Safety Management", funded by Korea Ministry of Environment (MOE) (Grant No. 2018002490006; 1485016918).

Institutional Review Board Statement: Not applicable.

Informed Consent Statement: Not applicable.

Data Availability Statement: Data is contained within the article or supplementary material.

Acknowledgments: J.K. acknowledges the support from the European Union's Horizon 2020 research and innovation programme (SABYDOMA Project under Grant Agreement No. 862296).

Conflicts of Interest: The authors declare no conflict of interest.

\section{References}

1. Sun, T.Y.; Gottschalk, F.; Hungerbühler, K.; Nowack, B. Comprehensive probabilistic modelling of environmental emissions of engineered nanomaterials. Environ. Pollut. 2014, 185, 69-76. [CrossRef] [PubMed]

2. Loewe, S.; Muischnek, H. Über Kombinationswirkungen I. Mitteilung: Hilfsmittel der Fragestellung. Naunyn-Schmiedebergs Arch. Exp. Pathol. Pharmakol. 1926, 114, 313-326. [CrossRef]

3. Kim, J.; Kim, S.; Schaumann, G.E. Reliable predictive computational toxicology methods for mixture toxicity: Toward the development of innovative integrated models for environmental risk assessment. Rev. Environ. Sci. Biotechnol. 2013, 12, 235-256. [CrossRef]

4. Bliss, C.I. The toxicity of poisons applied jointly 1. Ann. Appl. Biol. 1939, 26, 585-615. [CrossRef]

5. Gaudin, T.; Rotureau, P.; Fayet, G. Mixture Descriptors toward the Development of Quantitative Structure-Property Relationship Models for the Flash Points of Organic Mixtures. Ind. Eng. Chem. Res. 2015, 54, 6596-6604. [CrossRef]

6. Kar, S.; Ghosh, S.; Leszczynski, J. Single or mixture halogenated chemicals? Risk assessment and developmental toxicity prediction on zebrafish embryos based on weighted descriptors approach. Chemosphere 2018, 210, 588-596. [CrossRef]

7. Qin, L.T.; Chen, Y.H.; Zhang, X.; Mo, L.Y.; Zeng, H.H.; Liang, Y.P. QSAR prediction of additive and non-additive mixture toxicities of antibiotics and pesticide. Chemosphere 2018, 198, 122-129. [CrossRef]

8. Sobati, M.A.; Abooali, D.; Maghbooli, B.; Najafi, H. A new structure-based model for estimation of true critical volume of multi-component mixtures. Chemom. Intell. Lab. Syst. 2016, 155, 109-119. [CrossRef]

9. Wang, T.; Tang, L.; Luan, F.; Cordeiro, M.N.D.S. Prediction of the toxicity of binary mixtures by qsar approach using the hypothetical descriptors. Int. J. Mol. Sci. 2018, 19, 3423. [CrossRef] 
10. Mikolajczyk, A.; Sizochenko, N.; Mulkiewicz, E.; Malankowska, A.; Rasulev, B.; Puzyn, T. A chemoinformatics approach for the characterization of hybrid nanomaterials: Safer and efficient design perspective. Nanoscale 2019, 11, 11808-11818. [CrossRef]

11. Mikolajczyk, A.; Gajewicz, A.; Mulkiewicz, E.; Rasulev, B.; Marchelek, M.; Diak, M.; Hirano, S.; Zaleska-Medynska, A.; Puzyn, T. Nano-QSAR modeling for ecosafe design of heterogeneous $\mathrm{TiO}_{2}$-based nano-photocatalysts. Environ. Sci. Nano 2018, 5, 1150-1160. [CrossRef]

12. Mikolajczyk, A.; Malankowska, A.; Nowaczyk, G.; Gajewicz, A.; Hirano, S.; Jurga, S.; Zaleska-Medynska, A.; Puzyn, T. Combined experimental and computational approach to developing efficient photocatalysts based on $\mathrm{Au} / \mathrm{Pd}-\mathrm{TiO}_{2}$ nanoparticles. Environ. Sci. Nano 2016, 3, 1425-1435. [CrossRef]

13. Lopes, S.; Pinheiro, C.; Soares, A.M.V.M.; Loureiro, S. Joint toxicity prediction of nanoparticles and ionic counterparts: Simulating toxicity under a fate scenario. J. Hazard. Mater. 2016, 320, 1-9. [CrossRef]

14. Azevedo, S.L.; Holz, T.; Rodrigues, J.; Monteiro, T.; Costa, F.M.; Soares, A.M.V.M.; Loureiro, S. A mixture toxicity approach to predict the toxicity of Ag decorated ZnO nanomaterials. Sci. Total Environ. 2017, 579, 337-344. [CrossRef]

15. Wang, Z.; Zhang, F.; Wang, S.; Peijnenburg, W.J.G.M. Assessment and prediction of joint algal toxicity of binary mixtures of graphene and ionic liquids. Chemosphere 2017, 185, 681-689. [CrossRef]

16. Martín-de-Lucía, I.; Gonçalves, S.F.; Leganés, F.; Fernández-Piñas, F.; Rosal, R.; Loureiro, S. Combined toxicity of graphitediamond nanoparticles and thiabendazole to Daphnia magna. Sci. Total Environ. 2019, 688, 1145-1154. [CrossRef]

17. Wang, Z.; Wang, S.; Peijnenburg, W.J.G.M. Prediction of joint algal toxicity of nano- $\mathrm{CeO}_{2} / \mathrm{nano}^{-\mathrm{TiO}} 2$ and florfenicol: Independent action surpasses concentration addition. Chemosphere 2016, 156, 8-13. [CrossRef]

18. Ye, N.; Wang, Z.; Wang, S.; Peijnenburg, W.J.G.M. Toxicity of mixtures of zinc oxide and graphene oxide nanoparticles to aquatic organisms of different trophic level: Particles outperform dissolved ions. Nanotoxicology 2018, 12, 423-438. [CrossRef]

19. Altenburger, R.; Nendza, M.; Schuurmann, G. Mixture Toxicity and Its Modeling by Quantitative Structure-Activity Relationships. Environ. Toxicol. Chem. 2003, 22, 1900-1915. [CrossRef] [PubMed]

20. Raies, A.B.; Bajic, V.B. In silico toxicology: Computational methods for the prediction of chemical toxicity. Wiley Interdiscip. Rev. Comput. Mol. Sci. 2016, 6, 147-172. [CrossRef] [PubMed]

21. Cedergreen, N. Quantifying synergy: A systematic review of mixture toxicity studies within environmental toxicology. PLoS ONE 2014, 9, e96580. [CrossRef] [PubMed]

22. Naasz, S.; Altenburger, R.; Kühnel, D. Environmental mixtures of nanomaterials and chemicals: The Trojan-horse phenomenon and its relevance for ecotoxicity. Sci. Total Environ. 2018, 635, 1170-1181. [CrossRef] [PubMed]

23. Qu, R.; Wang, X.; Wang, Z.; Wei, Z.; Wang, L. Metal accumulation and antioxidant defenses in the freshwater fish Carassius auratus in response to single and combined exposure to cadmium and hydroxylated multi-walled carbon nanotubes. J. Hazard. Mater. 2014, 275, 89-98. [CrossRef]

24. Lorenz, C.S.; Wicht, A.J.; Guluzada, L.; Crone, B.; Karst, U.; Lee, H.J.; Triebskorn, R.; Haderlein, S.B.; Huhn, C.; Köhler, H.R. Nano-sized zeolites as modulators of thiacloprid toxicity on Chironomus riparius. PeerJ 2017, 2017, 1-19. [CrossRef] [PubMed]

25. $\mathrm{Hu}$, J.; Wang, D.; Forthaus, B.E.; Wang, J. Quantifying the effect of nanoparticles on $\mathrm{As}(\mathrm{V})$ ecotoxicity exemplified by nano-Fe ${ }_{2} \mathrm{O}_{3}$ (magnetic) and nano- $\mathrm{Al}_{2} \mathrm{O}_{3}$. Environ. Toxicol. Chem. 2012, 31, 2870-2876. [CrossRef]

26. Yu, Z.G.; Wang, W.X. Interaction of functionalized fullerenes and metal accumulation in Daphnia magna. Environ. Toxicol. Chem. 2014, 33, 1122-1128. [CrossRef]

27. Fayaz, A.M.; Balaji, K.; Girilal, M.; Yadav, R.; Kalaichelvan, P.T.; Venketesan, R. Biogenic synthesis of silver nanoparticles and their synergistic effect with antibiotics: A study against gram-positive and gram-negative bacteria. Nanomed. Nanotechnol. Biol. Med. 2010, 6, 103-109. [CrossRef]

28. Xia, X.; Li, Y.; Zhou, Z.; Feng, C. Bioavailability of adsorbed phenanthrene by black carbon and multi-walled carbon nanotubes to Agrobacterium. Chemosphere 2010, 78, 1329-1336. [CrossRef]

29. Liu, Y.; Wang, S.; Wang, Z.; Ye, N.; Fang, H.; Wang, D. $\mathrm{TiO}_{2}, \mathrm{SiO}_{2}$ and $\mathrm{ZrO}_{2}$ nanoparticles synergistically provoke cellular oxidative damage in freshwater microalgae. Nanomaterials 2018, 8, 95. [CrossRef]

30. Freitas, R.; Coppola, F.; De Marchi, L.; Codella, V.; Pretti, C.; Chiellini, F.; Morelli, A.; Polese, G.; Soares, A.M.V.M.; Figueira, E. The influence of Arsenic on the toxicity of carbon nanoparticles in bivalves. J. Hazard. Mater. 2018, 358, 484-493. [CrossRef]

31. Zhang, Z.; Yang, X.; Shen, M.; Yin, Y.; Liu, J. Sunlight-driven reduction of silver ion to silver nanoparticle by organic matter mitigates the acute toxicity of silver to Daphnia magna. J. Environ. Sci. 2015, 35, 62-68. [CrossRef]

32. Petersen, E.J.; Pinto, R.A.; Landrum, P.F.; Weber, W.J. Influence of carbon nanotubes on pyrene bioaccumulation from contaminated soils by earthworms. Environ. Sci. Technol. 2009, 43, 4181-4187. [CrossRef]

33. Völker, C.; Gräf, T.; Schneider, I.; Oetken, M.; Oehlmann, J. Combined effects of silver nanoparticles and $17 \alpha$-ethinylestradiol on the freshwater mudsnail Potamopyrgus antipodarum. Environ. Sci. Pollut. Res. 2014, 21, 10661-10670. [CrossRef]

34. Wang, X.; Qu, R.; Liu, J.; Wei, Z.; Wang, L.; Yang, S.; Huang, Q.; Wang, Z. Effect of different carbon nanotubes on cadmium toxicity to Daphnia magna: The role of catalyst impurities and adsorption capacity. Environ. Pollut. 2016, 208, 732-738. [CrossRef]

35. Iswarya, V.; Bhuvaneshwari, M.; Alex, S.A.; Iyer, S.; Chaudhuri, G.; Chandrasekaran, P.T.; Bhalerao, G.M.; Chakravarty, S.; Raichur, A.M.; Chandrasekaran, N.; et al. Combined toxicity of two crystalline phases (anatase and rutile) of Titania nanoparticles towards freshwater microalgae: Chlorella sp. Aquat. Toxicol. 2015, 161, 154-169. [CrossRef] [PubMed]

36. Henry, T.B.; Wileman, S.J.; Boran, H.; Sutton, P. Association of Hg2+ with aqueous (C60)n aggregates facilitates increased bioavailability of $\mathrm{Hg}^{2+}$ in zebrafish (Danio rerio). Environ. Sci. Technol. 2013, 47, 9997-10004. [CrossRef] [PubMed] 
37. Zhang, B.; Cho, M.; Fortner, J.D.; Lee, J.; Huang, C.H.; Hughes, J.B.; Kim, J.H. Delineating oxidative processes of aqueous C60 preparations: Role of THF peroxide. Environ. Sci. Technol. 2009, 43, 108-113. [CrossRef] [PubMed]

38. Hu, X.; Chen, Q.; Jiang, L.; Yu, Z.; Jiang, D.; Yin, D. Combined effects of titanium dioxide and humic acid on the bioaccumulation of cadmium in Zebrafish. Environ. Pollut. 2011, 159, 1151-1158. [CrossRef]

39. Dalai, S.; Pakrashi, S.; Bhuvaneshwari, M.; Iswarya, V.; Chandrasekaran, N.; Mukherjee, A. Toxic effect of Cr(VI) in presence of $\mathrm{n}-\mathrm{TiO}_{2}$ and $\mathrm{n}-\mathrm{Al}_{2} \mathrm{O}_{3}$ particles towards freshwater microalgae. Aquat. Toxicol. 2014, 146, 28-37. [CrossRef] [PubMed]

40. Yang, W.W.; Wang, Y.; Huang, B.; Wang, N.X.; Wei, Z.B.; Luo, J.; Miao, A.J.; Yang, L.Y. TiO2 nanoparticles act as a carrier of Cd bioaccumulation in the ciliate tetrahymena thermophila. Environ. Sci. Technol. 2014, 48, 7568-7575. [CrossRef]

41. Sun, H.; Ruan, Y.; Zhu, H.; Zhang, Z.; Zhang, Y.; Yu, L. Enhanced bioaccumulation of pentachlorophenol in carp in the presence of multi-walled carbon nanotubes. Environ. Sci. Pollut. Res. 2014, 21, 2865-2875. [CrossRef] [PubMed]

42. Srikanth, K.; Ahmad, I.; Rao, J.V.; Trindade, T.; Duarte, A.C.; Pereira, E. Modulation of glutathione and its dependent enzymes in gill cells of Anguilla anguilla exposed to silica coated iron oxide nanoparticles with or without mercury co-exposure under in vitro condition. Comp. Biochem. Physiol. C Toxicol. Pharmacol. 2014, 162, 7-14. [CrossRef] [PubMed]

43. Yang, W.W.; Miao, A.J.; Yang, L.Y. Cd ${ }^{2+}$ toxicity to a green alga Chlamydomonas reinhardtii as influenced by its adsorption on TiO2 engineered nanoparticles. PLOS ONE 2012, 7, e32300. [CrossRef] [PubMed]

44. Jimeno-Romero, A.; Oron, M.; Cajaraville, M.P.; Soto, M.; Marigómez, I. Nanoparticle size and combined toxicity of TiO 2 and DSLS (surfactant) contribute to lysosomal responses in digestive cells of mussels exposed to $\mathrm{TiO}_{2}$ nanoparticles. Nanotoxicology 2016, 10, 1168-1176. [CrossRef] [PubMed]

45. Kodali, V.K.; Roberts, J.R.; Shoeb, M.; Wolfarth, M.G.; Bishop, L.; Eye, T.; Barger, M.; Roach, K.A.; Friend, S.; Schwegler-Berry, D.; et al. Acute in vitro and in vivo toxicity of a commercial grade boron nitride nanotube mixture. Nanotoxicology 2017, 11, 1040-1058. [CrossRef]

46. Chen, C.; Unrine, J.M.; Judy, J.D.; Lewis, R.W.; Guo, J.; McNear, D.H.; Tsyusko, O.V. Toxicogenomic Responses of the Model Legume Medicago truncatula to Aged Biosolids Containing a Mixture of Nanomaterials $\left(\mathrm{TiO}_{2}, \mathrm{Ag}\right.$, and $\left.\mathrm{ZnO}\right)$ from a Pilot Wastewater Treatment Plant. Environ. Sci. Technol. 2015, 49, 8759-8768. [CrossRef]

47. Martins, A.d.C.; Azevedo, L.F.; de Souza Rocha, C.C.; Carneiro, M.F.H.; Venancio, V.P.; de Almeida, M.R.; Antunes, L.M.G.; de Carvalho Hott, R.; Rodrigues, J.L.; Ogunjimi, A.T.; et al. Evaluation of distribution, redox parameters, and genotoxicity in Wistar rats co-exposed to silver and titanium dioxide nanoparticles. J. Toxicol. Environ. Health Part A Curr. Issues 2017, 80, 1156-1165. [CrossRef]

48. Mohmood, I.; Ahmad, I.; Asim, M.; Costa, L.; Lopes, C.B.; Trindade, T.; Duarte, A.C.; Pereira, E. Interference of the co-exposure of mercury with silica-coated iron oxide nanoparticles can modulate genotoxicity induced by their individual exposures- $\mathrm{A}$ paradox depicted in fish under in vitro conditions. Environ. Sci. Pollut. Res. 2015, 22, 3687-3696. [CrossRef]

49. Judy, J.D.; McNear, D.H.; Chen, C.; Lewis, R.W.; Tsyusko, O.V.; Bertsch, P.M.; Rao, W.; Stegemeier, J.; Lowry, G.V.; McGrath, S.P.; et al. Nanomaterials in Biosolids Inhibit Nodulation, Shift Microbial Community Composition, and Result in Increased Metal Uptake Relative to Bulk/Dissolved Metals. Environ. Sci. Technol. 2015, 49, 8751-8758. [CrossRef]

50. Hartmann, N.B.; Von der Kammer, F.; Hofmann, T.; Baalousha, M.; Ottofuelling, S.; Baun, A. Algal testing of titanium dioxide nanoparticles-Testing considerations, inhibitory effects and modification of cadmium bioavailability. Toxicology 2010, 269, 190-197. [CrossRef]

51. Alabresm, A.; Mirshahghassemi, S.; Chandler, G.T.; Decho, A.W.; Lead, J. Use of PVP-coated magnetite nanoparticles to ameliorate oil toxicity to an estuarine meiobenthic copepod and stimulate the growth of oil-degrading bacteria. Environ. Sci. Nano 2017, 4, 1859-1865. [CrossRef]

52. Ginzburg, A.L.; Truong, L.; Tanguay, R.L.; Hutchison, J.E. Synergistic Toxicity Produced by Mixtures of Biocompatible Gold Nanoparticles and Widely Used Surfactants. ACS Nano 2018, 12, 5312-5322. [CrossRef] [PubMed]

53. Yu, Y.; Duan, J.; Li, Y.; Yu, Y.; Jin, M.; Li, C.; Wang, Y.; Sun, Z. Combined toxicity of amorphous silica nanoparticles and methylmercury to human lung epithelial cells. Ecotoxicol. Environ. Saf. 2015, 112, 144-152. [CrossRef] [PubMed]

54. Tourinho, P.S.; Waalewijn-Kool, P.L.; Zantkuijl, I.; Jurkschat, K.; Svendsen, C.; Soares, A.M.V.M.; Loureiro, S.; van Gestel, C.A.M. $\mathrm{CeO}_{2}$ nanoparticles induce no changes in phenanthrene toxicity to the soil organisms Porcellionides pruinosus and Folsomia candida. Ecotoxicol. Environ. Saf. 2015, 113, 201-206. [CrossRef]

55. Santaella, C.; Allainmat, B.; Simonet, F.; Chanéac, C.; Labille, J.; Auffan, M.; Rose, J.; Achouak, W. Aged TiO2-based nanocomposite used in sunscreens produces singlet oxygen under long-wave UV and sensitizes escherichia coli to cadmium. Environ. Sci. Technol. 2014, 48, 5245-5253. [CrossRef]

56. Henry, T.B.; Menn, F.M.; Fleming, J.T.; Wilgus, J.; Compton, R.N.; Sayler, G.S. Attributing effects of aqueous C60 nano-aggregates to tetrahydrofuran decomposition products in larval zebrafish by assessment of gene expression. Environ. Health Perspect. 2007, 115, 1059-1065. [CrossRef]

57. Wilke, C.M.; Tong, T.; Gaillard, J.F.; Gray, K.A. Attenuation of Microbial Stress Due to Nano-Ag and Nano-TiO2 Interactions under Dark Conditions. Environ. Sci. Technol. 2016, 50, 11302-11310. [CrossRef]

58. Huang, B.; Wei, Z.B.; Yang, L.Y.; Pan, K.; Miao, A.J. Combined Toxicity of Silver Nanoparticles with Hematite or Plastic Nanoparticles toward Two Freshwater Algae. Environ. Sci. Technol. 2019, 53, 3871-3879. [CrossRef]

59. Sanchís, J.; Olmos, M.; Vincent, P.; Farré, M.; Barceló, D. New Insights on the Influence of Organic Co-Contaminants on the Aquatic Toxicology of Carbon Nanomaterials. Environ. Sci. Technol. 2016, 50, 961-969. [CrossRef] 
60. Park, H.G.; Yeo, M.K. Comparison of gene expression patterns from zebrafish embryos between pure silver nanomaterial and mixed silver nanomaterial containing cells of Hydra magnipapillata. Mol. Cell. Toxicol. 2015, 11, 307-314. [CrossRef]

61. Hu, C.W.; Zhang, L.J.; Wang, W.L.; Cui, Y.B.; Li, M. Evaluation of the combined toxicity of multi-walled carbon nanotubes and sodium pentachlorophenate on the earthworm Eisenia fetida using avoidance bioassay and comet assay. Soil Biol. Biochem. 2014, 70, 123-130. [CrossRef]

62. Nigro, M.; Bernardeschi, M.; Costagliola, D.; Della Torre, C.; Frenzilli, G.; Guidi, P.; Lucchesi, P.; Mottola, F.; Santonastaso, M.; Scarcelli, V.; et al. $\mathrm{n}-\mathrm{TiO}_{2}$ and $\mathrm{CdCl}_{2}$ co-exposure to titanium dioxide nanoparticles and cadmium: Genomic, DNA and chromosomal damage evaluation in the marine fish European sea bass (Dicentrarchus labrax). Aquat. Toxicol. 2015, 168, 72-77. [CrossRef] [PubMed]

63. Hernández-Moreno, D.; Valdehita, A.; Conde, E.; Rucandio, I.; Navas, J.M.; Fernández-Cruz, M.L. Acute toxic effects caused by the co-exposure of nanoparticles of $\mathrm{ZnO}$ and $\mathrm{Cu}$ in rainbow trout. Sci. Total Environ. 2019, 687, 24-33. [CrossRef] [PubMed]

64. Zhang, L.; Hu, C.; Wang, W.; Ji, F.; Cui, Y.; Li, M. Acute toxicity of multi-walled carbon nanotubes, sodium pentachlorophenate, and their complex on earthworm Eisenia fetida. Ecotoxicol. Environ. Saf. 2014, 103, 29-35. [CrossRef] [PubMed]

65. Soler de la Vega, A.C.; Molins-Delgado, D.; Barceló, D.; Díaz-Cruz, M.S. Nanosized titanium dioxide UV filter increases mixture toxicity when combined with parabens. Ecotoxicol. Environ. Saf. 2019, 184, 109565. [CrossRef]

66. Naqvi, S.Z.H.; Kiran, U.; Ali, M.I.; Jamal, A.; Hameed, A.; Ahmed, S.; Ali, N. Combined efficacy of biologically synthesized silver nanoparticles and different antibiotics against multidrug-resistant bacteria. Int. J. Nanomed. 2013, 8, 3187-3195. [CrossRef]

67. Zindler, F.; Glomstad, B.; Altin, D.; Liu, J.; Jenssen, B.M.; Booth, A.M. Phenanthrene bioavailability and toxicity to Daphnia magna in the presence of carbon nanotubes with different physicochemical properties. Environ. Sci. Technol. 2016, 50, 12446-12454. [CrossRef]

68. Zou, X.Y.; Xu, B.; Yu, C.P.; Zhang, H.W. Combined toxicity of ferroferric oxide nanoparticles and arsenic to the ciliated protozoa Tetrahymena Pyriformis. Aquat. Toxicol. 2013, 134-135, 66-73. [CrossRef]

69. Zhai, Y.; Hunting, E.R.; Wouterse, M.; Peijnenburg, W.J.G.M.; Vijver, M.G. Importance of exposure dynamics of metal-based nano- $\mathrm{ZnO},-\mathrm{Cu}$ and $-\mathrm{Pb}$ governing the metabolic potential of soil bacterial communities. Ecotoxicol. Environ. Saf. 2017, 145, 349-358. [CrossRef]

70. Svartz, G.; Papa, M.; Gosatti, M.; Jordán, M.; Soldati, A.; Samter, P.; Guraya, M.M.; Pérez Coll, C.; Perez Catán, S. Monitoring the ecotoxicity of $\gamma-\mathrm{Al}_{2} \mathrm{O}_{3}$ and $\mathrm{Ni} / \gamma-\mathrm{Al}_{2} \mathrm{O}_{3}$ nanomaterials by means of a battery of bioassays. Ecotoxicol. Environ. Saf. 2017, 144, 200-207. [CrossRef]

71. Rossi, S.C.; Mela, M.; Boschen, S.L.; da Cunha, C.; Neto, F.F.; Ribeiro, C.A.O.; Neves, A.P.P.; Silva de Assis, H.C. Modulatory effect of nano $\mathrm{TiO}_{2}$ on $\mathrm{Pb}$ in Hoplias malabaricus trophically exposed. Environ. Toxicol. Pharmacol. 2014, 38, 71-78. [CrossRef] [PubMed]

72. Wang, Y.; Yuan, L.; Yao, C.; Ding, L.; Li, C.; Fang, J.; Sui, K.; Liu, Y.; Wu, M. A combined toxicity study of zinc oxide nanoparticles and vitamin $\mathrm{C}$ in food additives. Nanoscale 2014, 6, 15333-15342. [CrossRef] [PubMed]

73. Kim, K.T.; Klaine, S.J.; Lin, S.; Ke, P.C.; Kim, S.D. Acute toxicity of a mixture of copper and single-walled carbon nanotubes to Daphnia magna. Environ. Toxicol. Chem. 2010, 29, 122-126. [CrossRef] [PubMed]

74. Hua, J.; Peijnenburg, W.J.G.M.; Vijver, M.G. TiO2 nanoparticles reduce the effects of ZnO nanoparticles and Zn ions on zebrafish embryos (Danio rerio). NanoImpact 2016, 2, 45-53. [CrossRef]

75. Cao, Y.; Roursgaard, M.; Kermanizadeh, A.; Loft, S.; Møller, P. Synergistic effects of zinc oxide nanoparticles and fatty acids on toxicity to caco-2 cells. Int. J. Toxicol. 2015, 34, 67-76. [CrossRef]

76. Tsugita, M.; Morimoto, N.; Nakayama, M. SiO2 and TiO2 nanoparticles synergistically trigger macrophage inflammatory responses. Part. Fibre Toxicol. 2017, 14, 1-9. [CrossRef]

77. Pavagadhi, S.; Sathishkumar, M.; Balasubramanian, R. Uptake of $\mathrm{Ag}$ and $\mathrm{TiO}_{2}$ nanoparticles by zebrafish embryos in the presence of other contaminants in the aquatic environment. Water Res. 2014, 55, 280-291. [CrossRef]

78. Fan, W.; Peng, R.; Li, X.; Ren, J.; Liu, T.; Wang, X. Effect of titanium dioxide nanoparticles on copper toxicity to Daphnia magna in water: Role of organic matter. Water Res. 2016, 105, 129-137. [CrossRef]

79. Wang, C.; Liu, H.; Chen, J.; Tian, Y.; Shi, J.; Li, D.; Guo, C.; Ma, Q. Carboxylated multi-walled carbon nanotubes aggravated biochemical and subcellular damages in leaves of broad bean (Vicia faba L.) seedlings under combined stress of lead and cadmium. J. Hazard. Mater. 2014, 274, 404-412. [CrossRef]

80. Jośko, I.; Oleszczuk, P.; Pranagal, J.; Lehmann, J.; Xing, B.; Cornelissen, G. Effect of biochars, activated carbon and multiwalled carbon nanotubes on phytotoxicity of sediment contaminated by inorganic and organic pollutants. Ecol. Eng. 2013, 60, 50-59. [CrossRef]

81. Park, C.B.; Jung, J.W.; Yeom, D.H.; Jang, J.; Park, J.W.; Kim, Y.J. Interactive effects between components in binary mixtures of zinc sulfate and iron oxide nanoparticles on Daphnia magna. Mol. Cell. Toxicol. 2019, 15, 315-323. [CrossRef]

82. Anjum, N.A.; Srikanth, K.; Mohmood, I.; Sayeed, I.; Trindade, T.; Duarte, A.C.; Pereira, E.; Ahmad, I. Brain glutathione redox system significance for the control of silica-coated magnetite nanoparticles with or without mercury co-exposures mediated oxidative stress in European eel (Anguilla anguilla L.). Environ. Sci. Pollut. Res. 2014, 21, 7746-7756. [CrossRef] [PubMed]

83. Davarpanah, E.; Guilhermino, L. Are gold nanoparticles and microplastics mixtures more toxic to the marine microalgae Tetraselmis chuii than the substances individually? Ecotoxicol. Environ. Saf. 2019, 181, 60-68. [CrossRef] [PubMed] 
84. Wang, Q.; Chen, Q.; Zhou, P.; Li, W.; Wang, J.; Huang, C.; Wang, X.; Lin, K.; Zhou, B. Bioconcentration and metabolism of BDE-209 in the presence of titanium dioxide nanoparticles and impact on the thyroid endocrine system and neuronal development in zebrafish larvae. Nanotoxicology 2014, 8, 196-207. [CrossRef]

85. Renzi, M.; Blašković, A. Ecotoxicity of nano-metal oxides: A case study on Daphnia magna. Ecotoxicology 2019, 28, 878-889. [CrossRef]

86. Sundukov, Y.N. First record of the ground beetle Trechoblemus postilenatus (Coleoptera, Carabidae) in Primorskii krai. Far East. Entomol. 2006, 165, 16. [CrossRef]

87. Qiu, T.A.; Nguyen, T.H.T.; Hudson-Smith, N.V.; Clement, P.L.; Forester, D.C.; Frew, H.; Hang, M.N.; Murphy, C.J.; Hamers, R.J.; Feng, Z.V.; et al. Growth-Based Bacterial Viability Assay for Interference-Free and High-Throughput Toxicity Screening of Nanomaterials. Anal. Chem. 2017, 89, 2057-2064. [CrossRef]

88. Polak, N.; Read, D.S.; Jurkschat, K.; Matzke, M.; Kelly, F.J.; Spurgeon, D.J.; Stürzenbaum, S.R. Metalloproteins and phytochelatin synthase may confer protection against zinc oxide nanoparticle induced toxicity in Caenorhabditis elegans. Comp. Biochem. Physiol. C Toxicol. Pharmacol. 2014, 160, 75-85. [CrossRef]

89. Kim, I.; Lee, B.T.; Kim, H.A.; Kim, K.W.; Kim, S.D.; Hwang, Y.S. Citrate coated silver nanoparticles change heavy metal toxicities and bioaccumulation of Daphnia magna. Chemosphere 2016, 143, 99-105. [CrossRef]

90. Ogunsuyi, O.I.; Fadoju, O.M.; Akanni, O.O.; Alabi, O.A.; Alimba, C.G.; Cambier, S.; Eswara, S.; Gutleb, A.C.; Adaramoye, O.A.; Bakare, A.A. Genetic and systemic toxicity induced by silver and copper oxide nanoparticles, and their mixture in Clarias gariepinus (Burchell, 1822). Environ. Sci. Pollut. Res. 2019, 26, 27470-27481. [CrossRef]

91. Fang, T.; Watson, J.L.; Goodman, J.; Dimkpa, C.O.; Martineau, N.; Das, S.; McLean, J.E.; Britt, D.W.; Anderson, A.J. Does doping with aluminum alter the effects of $\mathrm{ZnO}$ nanoparticles on the metabolism of soil pseudomonads? Microbiol. Res. 2013, 168, 91-98. [CrossRef] [PubMed]

92. Canesi, L.; Frenzilli, G.; Balbi, T.; Bernardeschi, M.; Ciacci, C.; Corsolini, S.; Della, C.; Fabbri, R.; Faleri, C.; Focardi, S.; et al. Interactive effects of $\mathrm{n}-\mathrm{TiO}_{2}$ and 2,3,7,8-TCDD on the marine bivalve Mytilus galloprovincialis. Aquat. Toxicol. 2014, 153, 53-65. [CrossRef] [PubMed]

93. Deville, S.; Baré, B.; Piella, J.; Tirez, K.; Hoet, P.; Monopoli, M.P.; Dawson, K.A.; Puntes, V.F.; Nelissen, I. Interaction of gold nanoparticles and nickel(II) sulfate affects dendritic cell maturation. Nanotoxicology 2016, 10, 1395-1403. [CrossRef] [PubMed]

94. Della Torre, C.; Buonocore, F.; Frenzilli, G.; Corsolini, S.; Brunelli, A.; Guidi, P.; Kocan, A.; Mariottini, M.; Mottola, F.; Nigro, M.; et al. Influence of titanium dioxide nanoparticles on 2,3,7,8-tetrachlorodibenzo-p-dioxin bioconcentration and toxicity in the marine fish European sea bass (Dicentrarchus labrax). Environ. Pollut. 2015, 196, 185-193. [CrossRef]

95. Della Torre, C.; Balbi, T.; Grassi, G.; Frenzilli, G.; Bernardeschi, M.; Smerilli, A.; Guidi, P.; Canesi, L.; Nigro, M.; Monaci, F.; et al. Titanium dioxide nanoparticles modulate the toxicological response to cadmium in the gills of Mytilus galloprovincialis. J. Hazard. Mater. 2015, 297, 92-100. [CrossRef]

96. De La Rosa-García, S.C.; Martínez-Torres, P.; Gómez-Cornelio, S.; Corral-Aguado, M.A.; Quintana, P.; Gómez-Ortíz, N.M. Antifungal activity of $\mathrm{ZnO}$ and $\mathrm{MgO}$ nanomaterials and their mixtures against colletotrichum gloeosporioides strains from tropical fruit. J. Nanomater. 2018, 2018. [CrossRef]

97. Brausch, K.A.; Anderson, T.A.; Smith, P.N.; Maul, J.D. Effects of functionalized fullerenes on bifenthrin and tribufos toxicity to Daphnia magna: Survival, reproduction, and growth rate. Environ. Toxicol. Chem. 2010, 29, 2600-2606. [CrossRef]

98. Baun, A.; Sørensen, S.N.; Rasmussen, R.F.; Hartmann, N.B.; Koch, C.B. Toxicity and bioaccumulation of xenobiotic organic compounds in the presence of aqueous suspensions of aggregates of nano-C60. Aquat. Toxicol. 2008, 86, 379-387. [CrossRef]

99. Balbi, T.; Smerilli, A.; Fabbri, R.; Ciacci, C.; Montagna, M.; Grasselli, E.; Brunelli, A.; Pojana, G.; Marcomini, A.; Gallo, G.; et al. Co-exposure to $\mathrm{n}-\mathrm{TiO}_{2}$ and $\mathrm{Cd}^{2+}$ results in interactive effects on biomarker responses but not in increased toxicity in the marine bivalve M. galloprovincialis. Sci. Total Environ. 2014, 493, 355-364. [CrossRef]

100. Lyon, D.Y.; Adams, L.K.; Falkner, J.C.; Alvarez, P.J.J. Antibacterial activity of fullerene water suspensions: Effects of preparation method and particle size. Environ. Sci. Technol. 2006, 40, 4360-4366. [CrossRef]

101. Baek, M.J.; Son, J.; Park, J.; Seol, Y.; Sung, B.; Kim, Y.J. Quantitative prediction of mixture toxicity of $\mathrm{AgNO}_{3}$ and $\mathrm{ZnO}$ nanoparticles on Daphnia magna. Sci. Technol. Adv. Mater. 2020, 21, 333-345. [CrossRef] [PubMed]

102. Worms, I.A.M.; Boltzman, J.; Garcia, M.; Slaveykova, V.I. Cell-wall-dependent effect of carboxyl-CdSe/ZnS quantum dots on lead and copper availability to green microalgae. Environ. Pollut. 2012, 167, 27-33. [CrossRef] [PubMed]

103. Yan, J.; Lin, B.; Hu, C.; Zhang, H.; Lin, Z.; Xi, Z. The combined toxicological effects of titanium dioxide nanoparticles and bisphenol A on zebrafish embryos. Nanoscale Res. Lett. 2014, 9, 1-9. [CrossRef] [PubMed]

104. Hamdi, H.; De La Torre-Roche, R.; Hawthorne, J.; White, J.C. Impact of non-functionalized and amino-functionalized multiwall carbon nanotubes on pesticide uptake by lettuce (Lactuca sativa L.). Nanotoxicology 2014, 9, 172-180. [CrossRef] [PubMed]

105. Hu, C.; Cai, Y.; Wang, W.; Cui, Y.; Li, M. Toxicological effects of multi-walled carbon nanotubes adsorbed with nonylphenol on earthworm Eisenia fetida. Environ. Sci. Process. Impacts 2013, 15, 2125-2130. [CrossRef]

106. Fan, W.H.; Cui, M.M.; Shi, Z.W.; Tan, C.; Yang, X.P. Enhanced oxidative stress and physiological damage in Daphnia magna by copper in the presence of Nano-TiO 2 . J. Nanomater. 2012, 2012, 1-7. [CrossRef]

107. Fan, W.; Cui, M.; Liu, H.; Wang, C.; Shi, Z.; Tan, C.; Yang, X. Nano-TiO 2 enhances the toxicity of copper in natural water to Daphnia magna. Environ. Pollut. 2011, 159, 729-734. [CrossRef] 
108. Tian, S.Y.; Gao, Y.N.; Song, C.Z. Acute toxicities of penta-BDE in $\mathrm{TiO}_{2}$ nanoparticle suspensions to Daphnia magna. Adv. Mater. Res. 2014, 864-867, 261-265. [CrossRef]

109. Tan, C.; Wang, W.X. Modification of metal bioaccumulation and toxicity in Daphnia magna by titanium dioxide nanoparticles. Environ. Pollut. 2014, 186, 36-42. [CrossRef]

110. Seitz, F.; Bundschuh, M.; Dabrunz, A.; Bandow, N.; Schaumann, G.E.; Schulz, R. Titanium dioxide nanoparticles detoxify pirimicarb under UV irradiation at ambient intensities. Environ. Toxicol. Chem. 2012, 31, 518-523. [CrossRef]

111. Liu, Y.; Yan, Z.; Xia, J.; Wang, K.; Ling, X.; Yan, B. Potential toxicity in crucian carp following exposure to metallic nanoparticles of copper, chromium, and their mixtures: A comparative study. Polish J. Environ. Stud. 2017, 26, 2085-2094. [CrossRef]

112. Rosenfeldt, R.R.; Seitz, F.; Schulz, R.; Bundschuh, M. Heavy metal uptake and toxicity in the presence of titanium dioxide nanoparticles: A factorial approach using Daphnia magna. Environ. Sci. Technol. 2014, 48, 6965-6972. [CrossRef] [PubMed]

113. Rosenfeldt, R.R.; Seitz, F.; Senn, L.; Schilde, C.; Schulz, R.; Bundschuh, M. Nanosized titanium dioxide reduces copper toxicity-the role of organic material and the crystalline phase. Environ. Sci. Technol. 2015, 49, 1815-1822. [CrossRef] [PubMed]

114. Hartmann, N.B.; Legros, S.; Von der Kammer, F.; Hofmann, T.; Baun, A. The potential of $\mathrm{TiO}_{2}$ nanoparticles as carriers for cadmium uptake in Lumbriculus variegatus and Daphnia magna. Aquat. Toxicol. 2012, 118-119, 1-8. [CrossRef]

115. Wang, D.; Hu, J.; Irons, D.R.; Wang, J. Synergistic toxic effect of nano-TiO 2 and $\mathrm{As}(\mathrm{V})$ on Ceriodaphnia dubia. Sci. Total Environ. 2011, 409, 1351-1356. [CrossRef]

116. De La Torre-Roche, R.; Hawthorne, J.; Musante, C.; Xing, B.; Newman, L.A.; Ma, X.; White, J.C. Impact of Ag nanoparticle exposure on p, $\mathrm{p}^{\prime}$-DDE bioaccumulation by cucurbita pepo (Zucchini) and glycine max (Soybean). Environ. Sci. Technol. 2013, 47, 718-725. [CrossRef] [PubMed]

117. Park, C.B.; Jung, J.W.; Baek, M.; Sung, B.; Park, J.W.J.W.; Seol, Y.; Yeom, D.H.; Park, J.W.J.W.; Kim, Y.J. Mixture toxicity of metal oxide nanoparticles and silver ions on Daphnia magna. J. Nanoparticle Res. 2019, 21, 21. [CrossRef]

118. Zhang, X.; Sun, H.; Zhang, Z.; Niu, Q.; Chen, Y.; Crittenden, J.C. Enhanced bioaccumulation of cadmium in carp in the presence of titanium dioxide nanoparticles. Chemosphere 2007, 67, 160-166. [CrossRef]

119. Miao, W.; Zhu, B.; Xiao, X.; Li, Y.; Dirbaba, N.B.; Zhou, B.; Wu, H. Effects of titanium dioxide nanoparticles on lead bioconcentration and toxicity on thyroid endocrine system and neuronal development in zebrafish larvae. Aquat. Toxicol. 2015, 161, 117-126. [CrossRef]

120. Tong, T.; Wilke, C.M.; Wu, J.; Binh, C.T.T.; Kelly, J.J.; Gaillard, J.F.; Gray, K.A. Combined Toxicity of Nano-ZnO and Nano-TiO2: From Single- to Multinanomaterial Systems. Environ. Sci. Technol. 2015, 49, 8113-8123. [CrossRef]

121. Yi, X.; Zhang, K.; Han, G.; Yu, M.; Chi, T.; Jing, S.; Li, Z.; Zhan, J.; Wu, M. Toxic effect of triphenyltin in the presence of nano zinc oxide to marine copepod Tigriopus japonicus. Environ. Pollut. 2018, 243, 687-692. [CrossRef] [PubMed]

122. Tian, S.; Zhang, Y.; Song, C.; Zhu, X.; Xing, B. Titanium dioxide nanoparticles as carrier facilitate bioaccumulation of phenanthrene in marine bivalve, ark shell (Scapharca subcrenata). Environ. Pollut. 2014, 192, 59-64. [CrossRef] [PubMed]

123. Seo, Y.; Hwang, J.; Kim, J.; Jeong, Y.; Hwang, M.P.; Choi, J. Antibacterial activity and cytotoxicity of multi-walled carbon nanotubes decorated with silver nanoparticles. Int. J. Nanomed. 2014, 9, 4621-4629. [CrossRef]

124. Cui, X.Y.; Jia, F.; Chen, Y.X.; Gan, J. Influence of single-walled carbon nanotubes on microbial availability of phenanthrene in sediment. Ecotoxicology 2011, 20, 1277-1285. [CrossRef]

125. Rocco, L.; Santonastaso, M.; Nigro, M.; Mottola, F.; Costagliola, D.; Bernardeschi, M.; Guidi, P.; Lucchesi, P.; Scarcelli, V.; Corsi, I.; et al. Genomic and chromosomal damage in the marine mussel Mytilus galloprovincialis: Effects of the combined exposure to titanium dioxide nanoparticles and cadmium chloride. Mar. Environ. Res. 2015, 111, 144-148. [CrossRef] [PubMed]

126. Lammel, T.; Wassmur, B.; Mackevica, A.; Chen, C.E.L.; Sturve, J. Mixture toxicity effects and uptake of titanium dioxide (TiO 2 ) nanoparticles and 3,3",4,4"-tetrachlorobiphenyl (PCB77) in juvenile brown trout following co-exposure via the diet. Aquat. Toxicol. 2019, 213, 105195. [CrossRef]

127. Chen, F.; Wu, L.; Xiao, X.; Rong, L.; Li, M.; Zou, X. Mixture toxicity of zinc oxide nanoparticle and chemicals with different mode of action upon Vibrio fischeri. Environ. Sci. Eur. 2020, 32. [CrossRef]

128. Vannuccini, M.L.; Grassi, G.; Leaver, M.J.; Corsi, I. Combination effects of nano-TiO2 and 2,3,7,8-tetrachlorodibenzo-p-dioxin (TCDD) on biotransformation gene expression in the liver of European sea bass Dicentrarchus labrax. Comp. Biochem. Physiol. Part C Toxicol. Pharmacol. 2016, 176-177, 71-78. [CrossRef]

129. Shahverdi, A.R.; Fakhimi, A.; Shahverdi, H.R.; Minaian, S. Synthesis and effect of silver nanoparticles on the antibacterial activity of different antibiotics against Staphylococcus aureus and Escherichia coli. Nanomed. Nanotechnol. Biol. Med. 2007, 3 , 168-171. [CrossRef]

130. Gajbhiye, M.; Kesharwani, J.; Ingle, A.; Gade, A.; Rai, M. Fungus-mediated synthesis of silver nanoparticles and their activity against pathogenic fungi in combination with fluconazole. Nanomed. Nanotechnol. Biol. Med. 2009, 5, 382-386. [CrossRef]

131. Ferreira, J.L.R.; Lonné, M.N.; França, T.A.; Maximilla, N.R.; Lugokenski, T.H.; Costa, P.G.; Fillmann, G.; Antunes Soares, F.A.; de la Torre, F.R.; Monserrat, J.M. Co-exposure of the organic nanomaterial fullerene C60 with benzo[a]pyrene in Danio rerio (zebrafish) hepatocytes: Evidence of toxicological interactions. Aquat. Toxicol. 2014, 147, 76-83. [CrossRef] [PubMed]

132. Mahboob, S.; Al-Ghanim, K.A.; Al-Mulhim, N.M.A. Fish Exposure to Sub-Lethal Toxicity of Nano-Titanium Oxide and Changes in Muscular Antioxidant Enzymes and Protective Role of Vitamins C and E in Clarias gariepinus. Int. J. Agric. Biol. 2017, 19, 1505-1510. [CrossRef] 
133. Srivastava, S.; Kumar, A. Comparative cytotoxicity of nanoparticles and ions to Escherichia coli in binary mixtures. J. Environ. Sci. 2017, 55, 11-19. [CrossRef] [PubMed]

134. Li, P.; Li, J.; Wu, C.; Wu, Q.; Li, J. Synergistic antibacterial effects of $\beta$-lactam antibiotic combined with silver nanoparticles. Nanotechnology 2005, 16, 1912-1917. [CrossRef]

135. Kühnel, D.; Busch, W.; Meißner, T.; Springer, A.; Potthoff, A.; Richter, V.; Gelinsky, M.; Scholz, S.; Schirmer, K. Agglomeration of tungsten carbide nanoparticles in exposure medium does not prevent uptake and toxicity toward a rainbow trout gill cell line. Aquat. Toxicol. 2009, 93, 91-99. [CrossRef] [PubMed]

136. Kim, K.T.; Edgington, A.J.; Klaine, S.J.; Cho, J.W.; Kim, S.D. Influence of multiwalled carbon nanotubes dispersed in natural organic matter on speciation and bioavailability of copper. Environ. Sci. Technol. 2009, 43, 8979-8984. [CrossRef] [PubMed]

137. Chai, M.; Shi, F.; Li, R.; Liu, L.; Liu, Y.; Liu, F. Interactive effects of cadmium and carbon nanotubes on the growth and metal accumulation in a halophyte Spartina alterniflora (Poaceae). Plant Growth Regul. 2013, 71, 171-179. [CrossRef]

138. Cuahtecontzi-Delint, R.; Mendez-Rojas, M.A.; Bandala, E.R.; Quiroz, M.A.; Recillas, S.; Sanchez-Salas, J.L. Enhanced antibacterial activity of $\mathrm{CeO}_{2}$ nanoparticles by surfactants. Int. J. Chem. React. Eng. 2013, 11, 781-785. [CrossRef]

139. Kelsey, J.W.; White, J.C. Effect of C60 fullerenes on the accumulation of weathered p, $\mathrm{p}^{\prime}$-DDE by plant and earthworm species under single and multispecies conditions. Environ. Toxicol. Chem. 2013, 32, 1117-1123. [CrossRef]

140. Zhang, W.; Sun, X.; Chen, L.; Lin, K.F.; Dong, Q.X.; Huang, C.J.; Fu, R.B.; Zhu, J. Toxicological effect of joint cadmium selenium quantum dots and copper ion exposure on zebrafish. Environ. Toxicol. Chem. 2012, 31, 2117-2123. [CrossRef]

141. Schirinzi, G.F.; Pérez-Pomeda, I.; Sanchís, J.; Rossini, C.; Farré, M.; Barceló, D. Cytotoxic effects of commonly used nanomaterials and microplastics on cerebral and epithelial human cells. Environ. Res. 2017, 159, 579-587. [CrossRef] [PubMed]

142. Park, J.W.; Henry, T.B.; Menn, F.M.; Compton, R.N.; Sayler, G. No bioavailability of $17 \alpha$-ethinylestradiol when associated with nC60 aggregates during dietary exposure in adult male zebrafish (Danio rerio). Chemosphere 2010, 81, 1227-1232. [CrossRef] [PubMed]

143. Vale, G.; Franco, C.; Diniz, M.S.; dos Santos, M.M.C.; Domingos, R.F. Bioavailability of cadmium and biochemical responses on the freshwater bivalve Corbicula fluminea-The role of $\mathrm{TiO}_{2}$ nanoparticles. Ecotoxicol. Environ. Saf. 2014, 109, 161-168. [CrossRef] [PubMed]

144. Sun, H.; Zhang, X.; Niu, Q.; Chen, Y.; Crittenden, J.C. Enhanced accumulation of arsenate in carp in the presence of titanium dioxide nanoparticles. Water. Air. Soil Pollut. 2007, 178, 245-254. [CrossRef]

145. Al-Subiai, S.N.; Arlt, V.M.; Frickers, P.E.; Readman, J.W.; Stolpe, B.; Lead, J.R.; Moody, A.J.; Jha, A.N. Merging nano-genotoxicology with eco-genotoxicology: An integrated approach to determine interactive genotoxic and sub-lethal toxic effects of C 60 fullerenes and fluoranthene in marine mussels, Mytilus sp. Mutat. Res. Genet. Toxicol. Environ. Mutagen. 2012, 745, 92-103. [CrossRef] [PubMed]

146. Krysanov, E.Y.; Demidova, T.B. The effect of low concentrations of nanocrystalline cerium dioxide on the embryotoxicity of doxorubicin for fish. Dokl. Biol. Sci. 2012, 443, 117-119. [CrossRef]

147. Silveira, L.T.; Liberatore, A.M.A.; Koh, I.H.J.; Bizeto, M.A.; Camilo, F.F. Combined bactericidal activity of silver nanoparticles and hexadecylpyridinium salicylate ionic liquid. J. Nanoparticle Res. 2015, 17, 17. [CrossRef]

148. Wang, C.; Wei, Z.; Feng, M.; Wang, L.; Wang, Z. The effects of hydroxylated multiwalled carbon nanotubes on the toxicity of nickel to Daphnia magna under different pH levels. Environ. Toxicol. Chem. 2014, 33, 2522-2528. [CrossRef]

149. Han, Z.X.; He, G.D.; Wang, J.H.; Lv, C.X. Interaction influence of Cd(II) and nano- $\mathrm{TiO}_{2}$ on aggregation and adsorption kinetics toward marine algae. Int. J. Green Nanotechnol. Biomed. 2011, 3, 229-237. [CrossRef]

150. Glinski, A.; Liebel, S.; Pelletier, É.; Voigt, C.L.; Randi, M.A.F.; Campos, S.X.; Oliveira Ribeiro, C.A.; Filipak Neto, F. Toxicological interactions of silver nanoparticles and organochlorine pesticides in mouse peritoneal macrophages. Toxicol. Mech. Methods 2016, 26, 251-259. [CrossRef]

151. Judy, J.D.; Kirby, J.K.; McLaughlin, M.J.; McNear, D.; Bertsch, P.M. Symbiosis between nitrogen-fixing bacteria and Medicago truncatula is not significantly affected by silver and silver sulfide nanomaterials. Environ. Pollut. 2016, 214, 731-736. [CrossRef] [PubMed]

152. Farkas, J.; Bergum, S.; Nilsen, E.W.; Olsen, A.J.; Salaberria, I.; Ciesielski, T.M.; Baczek, T.; Konieczna, L.; Salvenmoser, W.; Jenssen, B.M. The impact of $\mathrm{TiO}_{2}$ nanoparticles on uptake and toxicity of benzo(a)pyrene in the blue mussel (Mytilus edulis). Sci. Total Environ. 2015, 511, 469-476. [CrossRef] [PubMed]

153. Fang, L.; Borggaard, O.K.; Holm, P.E.; Hansen, H.C.B.; Cedergreen, N. Toxicity and uptake of TRI- and dibutyltin in Daphnia magna in the absence and presence of nano-charcoal. Environ. Toxicol. Chem. 2011, 30, 2553-2561. [CrossRef] [PubMed]

154. Su, Y.; Yan, X.; Pu, Y.; Xiao, F.; Wang, D.; Yang, M. Risks of single-walled carbon nanotubes acting as contaminants-carriers: Potential release of phenanthrene in Japanese medaka (Oryzias latipes). Environ. Sci. Technol. 2013, 47, 4704-4710. [CrossRef] [PubMed]

155. Cui, X.; Wan, B.; Guo, L.H.; Yang, Y.; Ren, X. Insight into the mechanisms of combined toxicity of single-walled carbon nanotubes and nickel ions in macrophages: Role of P2X7 receptor. Environ. Sci. Technol. 2016, 50, 12473-12483. [CrossRef] [PubMed]

156. Wang, Y.; Peng, C.; Fang, H.; Sun, L.; Zhang, H.; Feng, J.; Duan, D.; Liu, T.; Shi, J. Mitigation of Cu(Ii) Phytotoxicity to Rice (Oryza sativa) in the Presence of $\mathrm{TiO}_{2}$ and $\mathrm{CeO}_{2}$ Nanoparticles Combined with Humic Acid. Environ. Toxicol. Chem. 2015, 34, 1588-1596. [CrossRef]

157. Hu, J.; Wang, D.; Wang, J.; Wang, J. Toxicity of lead on Ceriodaphnia dubia in the presence of nano-CeO ${ }_{2}$ and nano-TiO 2 . Chemosphere 2012, 89, 536-541. [CrossRef] 
158. Han, Z.; Li, J.; Bao, W.; Wang, J. Enhanced toxicity of atrazine to Daphnia magna in the presence of nano-CeO 2 . Chinese J. Geochemistry 2012, 31, 297-302. [CrossRef]

159. McShan, D.; Zhang, Y.; Deng, H.; Ray, P.C.; Yu, H. Synergistic Antibacterial Effect of Silver Nanoparticles Combined with Ineffective Antibiotics on Drug Resistant Salmonella typhimurium DT104. J. Environ. Sci. Health Part C Environ. Carcinog. Ecotoxicol. Rev. 2015, 33, 369-384. [CrossRef]

160. Fang, Q.; Shi, X.; Zhang, L.; Wang, Q.; Wang, X.; Guo, Y.; Zhou, B. Effect of titanium dioxide nanoparticles on the bioavailability, metabolism, and toxicity of pentachlorophenol in zebrafish larvae. J. Hazard. Mater. 2015, 283, 897-904. [CrossRef]

161. Hu, X.; Liu, J.; Zhou, Q.; Lu, S.; Liu, R.; Cui, L.; Yin, D.; Mayer, P.; Jiang, G. Bioavailability of organochlorine compounds in aqueous suspensions of fullerene: Evaluated with medaka (Oryzias latipes) and negligible depletion solid-phase microextraction. Chemosphere 2010, 80, 693-700. [CrossRef] [PubMed]

162. Yu, R.; Wu, J.; Liu, M.; Zhu, G.; Chen, L.; Chang, Y.; Lu, H. Toxicity of binary mixtures of metal oxide nanoparticles to Nitrosomonas europaea. Chemosphere 2016, 153, 187-197. [CrossRef] [PubMed]

163. Yang, W.W.; Li, Y.; Miao, A.J.; Yang, L.Y. Cd2+ toxicity as affected by bare $\mathrm{TiO}_{2}$ nanoparticles and their bulk counterpart. Ecotoxicol. Environ. Saf. 2012, 85, 44-51. [CrossRef] [PubMed]

164. Magesky, A.; Pelletier, É. Toxicity mechanisms of ionic silver and polymer-coated silver nanoparticles with interactions of functionalized carbon nanotubes on early development stages of sea urchin. Aquat. Toxicol. 2015, 167, 106-123. [CrossRef] [PubMed]

165. Shrestha, B.; Anderson, T.A.; Acosta-Martinez, V.; Payton, P.; Cañas-Carrell, J.E. The influence of multiwalled carbon nanotubes on polycyclic aromatic hydrocarbon (PAH) bioavailability and toxicity to soil microbial communities in alfalfa rhizosphere. Ecotoxicol. Environ. Saf. 2015, 116, 143-149. [CrossRef]

166. Yan, Z.; Liu, Y.; Sun, H.; Lu, G. Influence of multiwall carbon nanotubes on the toxicity of $17 \beta$-estradiol in the early life stages of zebrafish. Environ. Sci. Pollut. Res. 2018, 25, 7566-7574. [CrossRef]

167. Hernández-Moreno, D.; Li, L.; Connolly, M.; Conde, E.; Fernández, M.; Schuster, M.; Navas, J.M.; Fernández-Cruz, M.L. Mechanisms underlying the enhancement of toxicity caused by the coincubation of zinc oxide and copper nanoparticles in a fish hepatoma cell line. Environ. Toxicol. Chem. 2016, 35, 2562-2570. [CrossRef]

168. Schwab, F.; Bucheli, T.D.; Camenzuli, L.; Magrez, A.; Knauer, K.; Sigg, L.; Nowack, B. Diuron sorbed to carbon nanotubes exhibits enhanced toxicity to chlorella vulgaris. Environ. Sci. Technol. 2013, 47, 7012-7019. [CrossRef]

169. Lahive, E.; Matzke, M.; Durenkamp, M.; Lawlor, A.J.; Thacker, S.A.; Pereira, M.G.; Spurgeon, D.J.; Unrine, J.M.; Svendsen, C.; Lofts, S. Sewage sludge treated with metal nanomaterials inhibits earthworm reproduction more strongly than sludge treated with metal metals in bulk/salt forms. Environ. Sci. Nano 2017, 4, 78-88. [CrossRef]

170. Tang, Y.; Li, S.; Qiao, J.; Wang, H.; Li, L. Synergistic effects of nano-sized titanium dioxide and zinc on the photosynthetic capacity and survival of Anabaena sp. Int. J. Mol. Sci. 2013, 14, 14395-14407. [CrossRef] [PubMed]

171. Ma, X.; Wang, C. Fullerene nanoparticles affect the fate and uptake of trichloroethylene in phytoremediation systems. Environ. Eng. Sci. 2010, 27, 989-992. [CrossRef]

172. Hu, X.; Kang, J.; Lu, K.; Zhou, R.; Mu, L.; Zhou, Q. Graphene oxide amplifies the phytotoxicity of arsenic in wheat. Sci. Rep. 2014, 4, 1-10. [CrossRef] [PubMed]

173. Farkas, J.; Salaberria, I.; Styrishave, B.; Staňková, R.; Ciesielski, T.M.; Olsen, A.J.; Posch, W.; Flaten, T.P.; Krøkje, Å.; Salvenmoser, W.; et al. Exposure of juvenile turbot (Scophthalmus maximus) to silver nanoparticles and $17 \alpha$-ethinylestradiol mixtures: Implications for contaminant uptake and plasma steroid hormone levels. Environ. Pollut. 2017, 220, 328-336. [CrossRef]

174. Tan, C.; Fan, W.H.; Wang, W.X. Role of titanium dioxide nanoparticles in the elevated uptake and retention of cadmium and zinc in Daphnia magna. Environ. Sci. Technol. 2012, 46, 469-476. [CrossRef] [PubMed]

175. Shen, M.; Xia, X.; Zhai, Y.; Zhang, X.; Zhao, X.; Zhang, P. Influence of carbon nanotubes with preloaded and coexisting dissolved organic matter on the bioaccumulation of polycyclic aromatic hydrocarbons to Chironomus plumosus larvae in sediment. Environ. Toxicol. Chem. 2014, 33, 182-189. [CrossRef] [PubMed]

176. Azevedo Costa, C.L.; Chaves, I.S.; Ventura-Lima, J.; Ferreira, J.L.R.; Ferraz, L.; De Carvalho, L.M.H.; Monserrat, J.M. In vitro evaluation of co-exposure of arsenium and an organic nanomaterial (fullerene, C60) in zebrafish hepatocytes. Comp. Biochem. Physiol. C Toxicol. Pharmacol. 2012, 155, 206-212. [CrossRef] [PubMed]

177. Yan, X.M.; Zha, J.M.; Shi, B.Y.; Wang, D.S.; Wang, Z.J.; Tang, H.X. In vivo toxicity of nano-C60 aggregates complex with atrazine to aquatic organisms. Chin. Sci. Bull. 2010, 55, 339-345. [CrossRef]

178. Cano, A.M.; Maul, J.D.; Saed, M.; Irin, F.; Shah, S.A.; Green, M.J.; French, A.D.; Klein, D.M.; Crago, J.; Canas-Carrell, J.E. Trophic Transfer and Accumulation of Multiwalled Carbon Nanotubes in the Presence of Copper Ions in Daphnia magna and Fathead Minnow (Pimephales promelas). Environ. Sci. Technol. 2018, 52, 794-800. [CrossRef]

179. Nunes, S.M.; Josende, M.E.; Ruas, C.P.; Gelesky, M.A.; Júnior, F.M.R.d.S.; Fattorini, D.; Regoli, F.; Monserrat, J.M.; Ventura-Lima, J. Biochemical responses induced by co-exposition to arsenic and titanium dioxide nanoparticles in the estuarine polychaete Laeonereis acuta. Toxicology 2017, 376, 51-58. [CrossRef]

180. Ko, K.S.; Koh, D.C.; Kong, I.C. Toxicity evaluation of individual and mixtures of nanoparticles based on algal chlorophyll content and cell count. Materials 2018, 11, 121. [CrossRef]

181. Gao, M.; Zhang, Z.; Lv, M.; Song, W.; Lv, Y. Toxic effects of nanomaterial-adsorbed cadmium on Daphnia magna. Ecotoxicol. Environ. Saf. 2018, 148, 261-268. [CrossRef] [PubMed] 
182. Yu, R.; Wu, J.; Liu, M.; Chen, L.; Zhu, G.; Lu, H. Physiological and transcriptional responses of Nitrosomonas europaea to TiO2 and ZnO nanoparticles and their mixtures. Environ. Sci. Pollut. Res. 2016, 23, 13023-13034. [CrossRef] [PubMed]

183. Sun, H.; Zhang, X.; Zhang, Z.; Chen, Y.; Crittenden, J.C. Influence of titanium dioxide nanoparticles on speciation and bioavailability of arsenite. Environ. Pollut. 2009, 157, 1165-1170. [CrossRef] [PubMed]

184. Molins-Delgado, D.; Gago-Ferrero, P.; Díaz-Cruz, M.S.; Barceló, D. Single and joint ecotoxicity data estimation of organic UV filters and nanomaterials toward selected aquatic organisms. Urban groundwater risk assessment. Environ. Res. 2016, 145, 126-134. [CrossRef]

185. Ye, N.; Wang, Z.; Fang, H.; Wang, S.; Zhang, F. Combined ecotoxicity of binary zinc oxide and copper oxide nanoparticles to Scenedesmus obliquus. J. Environ. Sci. Health Part A Toxic/Hazardous Subst. Environ. Eng. 2017, 52, 555-560. [CrossRef]

186. Liu, Y.; Baas, J.; Peijnenburg, W.J.G.M.; Vijver, M.G. Evaluating the Combined Toxicity of Cu and ZnO Nanoparticles: Utility of the Concept of Additivity and a Nested Experimental Design. Environ. Sci. Technol. 2016, 50, 5328-5337. [CrossRef]

187. Rosenfeldt, R.R.; Seitz, F.; Zubrod, J.P.; Feckler, A.; Merkel, T.; Lüderwald, S.; Bundschuh, R.; Schulz, R.; Bundschuh, M. Does the presence of titanium dioxide nanoparticles reduce copper toxicity? A factorial approach with the benthic amphipod Gammarus fossarum. Aquat. Toxicol. 2015, 165, 154-159. [CrossRef]

188. Jośko, I.; Oleszczuk, P.; Skwarek, E. Toxicity of combined mixtures of nanoparticles to plants. J. Hazard. Mater. 2017, 331, 200-209. [CrossRef]

189. Yang, X.Y.; Edelmann, R.E.; Oris, J.T. Suspended C60 nanoparticles protect against short-term UV and fluoranthene photo-induced toxicity, but cause long-term cellular damage in Daphnia magna. Aquat. Toxicol. 2010, 100, 202-210. [CrossRef]

190. Park, J.W.; Henry, T.B.; Ard, S.; Menn, F.M.; Compton, R.N.; Sayler, G.S. The association between nC 60 and $17 \alpha-$ ethinylestradiol (EE2) decreases EE2 bioavailability in zebrafish and alters nanoaggregate characteristics. Nanotoxicology 2011, 5, 406-416. [CrossRef]

191. Xia, X.; Chen, X.; Zhao, X.; Chen, H.; Shen, M. Effects of carbon nanotubes, chars, and ash on bioaccumulation of perfluorochemicals by chironomus plumosus larvae in sediment. Environ. Sci. Technol. 2012, 46, 12467-12475. [CrossRef] [PubMed]

192. Shen, M.; Xia, X.; Wang, F.; Zhang, P.; Zhao, X. Influences of multiwalled carbon nanotubes and plant residue chars on bioaccumulation of polycyclic aromatic hydrocarbons by Chironomus plumosus larvae in sediment. Environ. Toxicol. Chem. 2012, 31, 202-209. [CrossRef] [PubMed]

193. Tao, X.; He, Y.; Fortner, J.D.; Chen, Y.; Hughes, J.B. Effects of aqueous stable fullerene nanocrystal (nC60) on copper (trace necessary nutrient metal): Enhanced toxicity and accumulation of copper in Daphnia magna. Chemosphere 2013, 92, 1245-1252. [CrossRef]

194. Meng, Y.; Wang, S.; Wang, Z.; Ye, N.; Fang, H. Algal toxicity of binary mixtures of zinc oxide nanoparticles and tetrabromobisphenol A: Roles of dissolved organic matters. Environ. Toxicol. Pharmacol. 2018, 64, 78-85. [CrossRef] [PubMed]

195. Ferguson, P.L.; Chandler, G.T.; Templeton, R.C.; Demarco, A.; Scrivens, W.A.; Englehart, B.A. Influence of sediment-Amendment with single-walled carbon nanotubes and diesel soot on bioaccumulation of hydrophobic organic contaminants by benthic invertebrates. Environ. Sci. Technol. 2008, 42, 3879-3885. [CrossRef] [PubMed]

196. Zhu, X.; Zhou, J.; Cai, Z. TiO2 nanoparticles in the marine environment: Impact on the toxicity of tributyltin to abalone (Haliotis diversicolor supertexta) embryos. Environ. Sci. Technol. 2011, 45, 3753-3758. [CrossRef] [PubMed]

197. Yu, Z.G.; Wang, W.X. Influences of ambient carbon nanotubes on toxic metals accumulation in Daphnia magna. Water Res. 2013, 47, 4179-4187. [CrossRef] [PubMed]

198. Trinh, X.T.; Choi, J.S.; Jeon, H.; Byun, H.G.; Yoon, T.H.; Kim, J. Quasi-SMILES-Based Nano-Quantitative Structure-Activity Relationship Model to Predict the Cytotoxicity of Multiwalled Carbon Nanotubes to Human Lung Cells. Chem. Res. Toxicol. 2018, 31, 183-190. [CrossRef]

199. Choi, J.-S.; Ha, K.M.; Trinh, X.T.; Yoon, T.H.; Byun, H.G. Towards a generalized toxicity prediction model for metal oxide nanomaterials using integrated data from different sources. Sci. Rep. 2018, 8, 6110. [CrossRef]

200. Choi, J.S.; Trinh, T.X.; Yoon, T.H.; Kim, J.; Byun, H.G. Quasi-QSAR for predicting the cell viability of human lung and skin cells exposed to different metal oxide nanomaterials. Chemosphere 2019, 217, 243-249. [CrossRef]

201. Oh, E.; Liu, R.; Nel, A.; Gemill, K.B.; Bilal, M.; Cohen, Y.; Medintz, I.L. Meta-analysis of cellular toxicity for cadmium-containing quantum dots. Nat. Nanotechnol. 2016, 11, 479-486. [CrossRef] [PubMed]

202. Trinh, T.X.; Ha, M.K.; Choi, J.S.; Byun, H.G.; Yoon, T.H. Curation of datasets, assessment of their quality and completeness, and nanoSAR classification model development for metallic nanoparticles. Environ. Sci. Nano 2018, 5, 1902-1910. [CrossRef]

203. Burk, J.; Sikk, L.; Burk, P.; Manshian, B.B.; Soenen, S.J.; Scott-Fordsmand, J.J.; Tamm, T.; Tämm, K. Fe-Doped ZnO nanoparticle toxicity: Assessment by a new generation of nanodescriptors. Nanoscale 2018, 10, 21985-21993. [CrossRef] [PubMed]

204. Tämm, K.; Sikk, L.; Burk, J.; Rallo, R.; Pokhrel, S.; Mädler, L.; Scott-Fordsmand, J.J.; Burk, P.; Tamm, T. Parametrization of nanoparticles: Development of full-particle nanodescriptors. Nanoscale 2016, 8, 16243-16250. [CrossRef] [PubMed]

205. Économiques, O. De Coopération et de Développement Guidance Document on the Validation of (Quantitative) Structure-Activity Relationship [(Q)Sar] Models; OECD: Paris, France, 2007; Volume 2, pp. 1-154. [CrossRef]

206. Raunio, H. In silico toxicology non-testing methods. Front. Pharmacol. 2011, 2, 1-8. [CrossRef]

207. Économiques, O. De Coopération et de Développement Test No. 211: Daphnia magna Reproduction Test; OECD: Paris, France, 2012. [CrossRef]

208. Économiques, O. De Coopération et de Développement Test No. 236: Fish Embryo Acute Toxicity (FET) Test; OECD: Paris, France, 2013; pp. 1-22. [CrossRef] 
209. OECD Test Guideline No. 212: Fish, Short-term Toxicity Test on Embryo and Sac-fry Stages. Organisation for Economic Cooperation and Development; OECD: Paris, France, 1998; Volume 212, pp. 1-20. [CrossRef]

210. Vinken, M. Omics-based input and output in the development and use of adverse outcome pathways. Curr. Opin. Toxicol. 2019, 18, 8-12. [CrossRef] 